Neuropsychobiology

The abstracts are only available online, free of charge, at www.karger.com/doi/10.1159/000230666

Annual Conference of the Swiss Society of Sleep Research, Sleep Medicine and Chronobiology (SSSSC) and the Swiss Society of Biological Psychiatry (SSBP)

\title{
Sleepless Mind. Mindless Sleep?
}

Bern, March 25 and 26, 2009

\section{Abstracts of the SSSSC}

Chairmen (SSSSC)

Christian Cajochen, Basel

Johannes Mathis, Bern

\section{Contents}

Oral Presentations

Abstracts 1-7

Poster Presentations

Abstracts 8-46

Author Index 


\section{Oral Presentations}

1

\section{No Rescue of the Impaired Homeostasic Sleep Rebound in DBA/2J Mice with Attentuation of the Stress Response}

\author{
V. Mongrain', T. Curie1, P. Gip², H.C. Heller², P. Franken ${ }^{1,2}$ \\ ${ }^{1} \mathrm{CIG}$, University of Lausanne, Lausanne, Switzerland, \\ ${ }^{2}$ Stanford University, Stanford, Calif., USA
}

Introduction: We have previously shown that both the change in EEG delta power and in clock-gene expression after sleep deprivation (SD) in mice depends on genetic background. Among several inbred strains, DBA/2J (D2) mice showed the smallest increase in delta power and the largest changes in clockgenes expression in the forebrain [1]. SD in rodents induces a stress response as it increases corticosterone secretion [2,3]. This study aimed at determining the contribution of changes in corticosterone to the homeostatic response of EEG delta power and clock-gene expression. Methods: Experiment 1: Mice from C57BL/6J, AKR/J, and D2 inbred strains were submitted to SD by gentle handling (ZT0-6) and killed immediately after for plasma corticosterone measurements. Experiment 2: Sleep of adrenalectomized (ADX) and sham-lesioned (sham) D2 mice was recorded for a baseline day and during recovery from SD, before and after surgery. Experiment 3: ADX and sham D2 mice were submitted to either baseline condition or SD and killed at ZT6 when brains were collected for analysis of forebrain clockgene expression using qPCR. Results: SD induced a higher increase in corticosterone secretion in D2 mice compared to the other two strains. Nevertheless, the delta power rebound after $\mathrm{SD}$ was similar in ADX and sham D2 mice. ADX attenuated the SD-mediated increase in mPer1-3 expression but did not change the SD-dependant decrease in mDbp mRNA. Discussion: While the larger SD-mediated increase in corticosterone observed in D2 mice could partly explain the larger increase in mPer expression in this strain, a higher stress response seems not to underlie the lower EEG delta power rebound after SD in this strain. The findings also indicate that stress does not importantly contribute to the homeostatic regulation of EEG delta power.

\section{References}

1. Franken P, Thomason R, Heller HC, O'hara BF: A non-circadian role for clock-genes in sleep homeostasis: a strain comparison. BMC Neurosci 2007; 8:87.

2. Hairston IS, Ruby NF, Brooke S, Peyron C, Denning DP, Heller HC, Sapolsky RM: Sleep deprivation elevates plasma corticosterone levels in neonatal rats. Neurosci Lett 2001;315:29-32.
3. Tobler I, Murison R, Ursin R, Ursin H, Borbély AA: The effects of sleep deprivation and recovery on plasma corticosterone in the rat. Neurosci Lett 1983;35:297-300.

Research supported by the University of Lausanne, the NIH (MH67752), FNS (3100A0-111974), and an NSERC fellowship.

\section{2 \\ Increase in Slow Wave Sleep During Multiple Naps in Women with Major Depression: Sleep on Higher Sleep Pressure?}

\section{S. Frey, A. Birchler-Pedross, P. Brunner, T. Götz,} V. Knoblauch, C. Cajochen

Centre for Chronobiology, Psychiatric University Clinics, Basel, Switzerland

Homeostatic sleewake regulation (Process S) has been hypothesized to be impaired in depression. It is not yet clear whether the saturation of process $\mathrm{S}$ during wakefulness or its decline rate during sleep or both may be altered in depression. In an ongoing study, we aim at quantifying process $\mathrm{S}$ in women suffering from major depression and healthy controls during short sleewake cycles under constant routine conditions. Eight females with major depression (mean age $23 \mathrm{y}, \mathrm{SD}+/-4.12 \mathrm{y}$ ) and nine healthy young females (mean age 25.3, SD +/- 4.03y) followed a protocol over 40 -h with 10 short sleewake cycles ("nap protocol") in a chronobiology facility under constant light and temperature conditions. Polysomnographic recordings were carried out continuously (12 EEGs, EOG, EMG). Additionally, subjective sleepiness was assessed by the Karolinska Sleepiness Scale. Slow wave sleep, as a marker for the decrease of process S, and subjective sleepiness were each subjected to a repeated twway mixed ANOVA with the factors "group" (depressed vs. healthy subjects) and "nap" (sleep episodes) or "time" (for sleepiness ratings) respectively. Results disclosed a main effect of the factors "nap" ( $<<0.0001$, "group" ( $p<0.01)$, and an interaction effect of "nap x group" $(\mathrm{p}<0.05)$ on slow wave sleep. Post-hoc analysis revealed that women with major depression had significantly more slow wave sleep in naps which occurred in the evening during the wake maintenance zone $(p<0.05)$. Results for subjective sleepiness showed a main effect of the factors "session" $(p<0.001)$ and "group" $(p<0.01)$ whereas no interaction effect "session x group" was observed. Post-hoc analysis on subjective sleepiness ratings yielded significantly higher scores for almost all time points during the 40-h nap protocol for women with major depression compared to healthy women. Our data indicate that women with major depression sleep on a higher sleep pressure level despite the fact that multiple naps encourage low sleep pressure conditions. This can be explained by either a faster

\begin{tabular}{ll}
\hline KARGER & (c) 2009 S. Karger AG, Basel \\
0302-282X/09/0594-0246\$26.00/0 \\
$\begin{array}{l}\text { Fax +41 61 306 1234 } \\
\begin{array}{l}\text { E-Mail karger@karger.ch } \\
\text { www.karger.com }\end{array}\end{array}$ & $\begin{array}{l}\text { Accessible online at: } \\
\text { www.karger.com/nps }\end{array}$
\end{tabular}


buildup of process $\mathrm{S}$ or a weaker circadian alerting signal, particularly during the wake maintenance zone in depressed women.

Research supported by the Swiss National Science Foundation

\section{3}

\section{Effects of a 24h Light-Dark Rhythm in Neonatal Care on Sleep and Crying Pattern of Preterm Infants}

\author{
C. Guyer', J. Fontijn'2, R. Huber'1, H.U. Bucher², O.G. Jenni \\ ${ }^{1}$ Child Development Center, University Children's Hospital \\ Zurich, ${ }^{2}$ Department Of Neonatology, University Hospital \\ Zurich, Switzerland
}

Aims: The influence of a $24 \mathrm{~h}$ light-dark rhythm in neonatal care on sleep and crying patterns of preterm infants was examined, using standard diary measures over 3 consecutive days and actigraphy data at corrected ages of 5,11 and 25 weeks. Methods: 41 preterm infants ( $<32$ weeks gestational age) were either nursed in the standard $24 \mathrm{~h}$ dim light condition (control group; $\mathrm{n}=21$ ) or in a cycled light condition (7am-7pm light, 7pm-7am dark; intervention group; $\mathrm{n}=20$ ). Sleecrying diary at 5 and 11 weeks corrected age were analysed in four $6 \mathrm{~h}$ intervals starting at midnight, by following variables: sleep, wake and content, fussing, crying and unsoothable crying. Actigraphy data were analysed at all three time points using periodogramm analysis. Furthermore an overall control group of 19 term infants were included, using the same methodological and analytical measures. Results: Sleecrying diary: Using repeated measure ANOVA fussing and crying showed an age (fussing: $\mathrm{P}<0.008$, crying: $\mathrm{P}<0.00$ ) and interval (fussing: $\mathrm{P}<0.00$, crying: $\mathrm{P}<0.001$ ) effect with more fussing and crying at 5 weeks and in the evening hours. Furthermore crying revealed an interaction between age, interval and intervention. (Cycled condition: less crying between 6:00 - 12:00 at 5 weeks and between 6:00 - 18:00 at 11 weeks of age; $\mathrm{P}<0.021$ ). Actigraphy data: Repeated measure ANOVA showed a steadily increase in $24 \mathrm{~h}$ day-night rhythm with age $(\mathrm{P}<0.00)$. No group effect in the preterm infants could be seen, but in comparison with term infants, preterm infants showed an earlier and stronger $24 \mathrm{~h}$ day-night rhtyhm. Summary and Conclusion: Nursing in a cycled light condition reduced crying episodes at certain times of day at 5 and 11 weeks corrected age, detected by sleecrying diary. Entrainment of a $24 \mathrm{~h}$ day-night rhythm could not be accelerated by cycled light. Preterm infants showed an earlier and stronger 24 h day-night rhythm than term infants.

\section{4}

\section{EEG Spectra in Healthy Older Adults Across All Circadian Phases During NREM Sleep}

\author{
M. Münch ${ }^{1,2}$, E.J. Silva', J.M. Ronda ${ }^{1}$, C.A. Czeisler', \\ J.F. Duffy ${ }^{1}$
}

'Division of Sleep Medicine, Brigham \& Women's Hospital, Harvard Medical School Boston, Mass., USA, ${ }^{2}$ Current

Address: Swiss Federal Institute of Technology Lausanne, Laboratory for Solar Energy and Building Physics,

Lausanne, Switzerland

Healthy aging is associated with changes in sleewake regulation, and those changes often lead to problems sleeping, both during the night and during daytime. The aim of our study was to examine the EEG sleep spectra during non-rapid eye movement (NREM) sleep when sleep was scheduled at all times of day. Twenty-four healthy older subjects $(64.2 \pm 2.3 \mathrm{yrs} ; 13 \mathrm{f}, 11$ $\mathrm{m})$ participated in an inpatient protocol. After 3 baseline (BL) days, they were scheduled to live on 20-h "days" consisting of $6.7 \mathrm{~h}$ of bed rest and $13.3 \mathrm{~h}$ of wakefulness for 12 consecutive days (forced desynchrony, FD). The electroencephalogram (EEG) was recorded during all scheduled sleep episodes, and all EEGs were visually scored and subjected to spectral analysis. Compared to BL nights, EEG activity in the slow-wave $(0.5-5.25 \mathrm{~Hz})$, theta (6-6.25, $7 \mathrm{~Hz}$ ), alpha (10-11.25 Hz) and high spindle range (14.5$15.5 \mathrm{~Hz}$ ) was significantly greater during FD, when subjects slept across many times of day. During FD, there was a significant interaction between homeostatic and circadian dependent factors such that EEG delta activity $(0.5-1.5 \mathrm{~Hz})$ was higher in the biological morning/early afternoon than at other times of day. EEG activity was significantly increased in almost all frequency ranges $(0.5-20.5 \mathrm{~Hz})$ during the biological day, when endogenous melatonin was not present, except for the lower EEG spindle range (12.25-14 Hz). Our results show age-related changes in the sleep EEG spectra in healthy older subjects and provide evidence for why it is more difficult for older adults to maintain consolidated sleep, especially when sleep occurs during the daytime.

This study was supported by NIH grant P01 AG09975 (to C.A.C.) and was conducted in the BWH GCRC supported by M01 RR02635; M.M. was supported by fellowships from the Novartis Foundation (Switzerland), the W. \& T. La-Roche Foundation (Switzerland), and Jazz Pharmaceuticals (Palo Alto, CA, USA)

\section{5}

\section{Mechanisms Underlying the Effects of GABAA Sedative-Hypnotic Drugs}

\section{R. Winsky-Sommerer \\ Institute of Pharmacology and Toxicology, University of Zurich, Switzerland}

The predominant inhibitory transmitter, gamma-aminobutyric acid (GABA), and its GABAA receptors play an important role in the neuronal systems regulating sleep. In particular, the most 
effective hypnotics, benzodiazepines (BZ) and BZ-like compounds, target GABAA receptors. However, many hypnotics display side-effects. Therefore, there is a need to develop new compounds to treat insomnia. Interestingly, the effects of GABAA receptor agonists on sleep are substantially different from those evoked by BZ or BZ-like compounds. One of our aims is to decipher in mice the mechanisms underlying the specific effects of GABAA agonists, e.g. THIP/Gaboxadol and muscimol, on behavior and the electroencephalogram (EEG). Traditional hypnotics target synaptic GABAA receptors mediating "phasic" inhibition. We were able to demonstrate in vivo that THIP acts at extrasynaptic GABAA receptors mediating a non-desensitizing "tonic" inhibition in the brain. In addition, we demonstrated a relationship between regional electroencephalogram synchronization and the alteration of behavior induced by muscimol. Understanding the mechanisms underlying physiological and "pharmacological" sleep could provide a better basis to develop new compounds to treat sleep disorders, as well as to characterize their effects on the EEG and performance in human subjects.

Forschungskredit from the University of Zurich;EU MCMCRTN-CT-2004512362 .

\section{6}

\section{Adverse Health Effects of Altitude on Untreated Patients with Obstructive Sleep Apnea. A Randomized, Controlled Study}

\author{
Y. Nussbaumer ${ }^{1}$, N. Schuepfer ${ }^{2}$, S. Ulrich ${ }^{1}$, K.E. Bloch ${ }^{1}$ \\ ${ }^{1}$ Pulmonary Division, University Hospital, Zurich, ${ }^{2}$ Institute \\ of human movement sciences and sport, ETH Zurich, \\ Switzerland
}

Background: Many patients with obstructive sleep apnea syndrome (OSA) travel to the mountains for recreational and professional activities while temporarily discontinuing CPAP. Since the physiologic consequences of altitude exposure in OSA patients are not known, we evaluated the hypothesis that sleep related breathing disturbances are aggravated and cardiovascular stress is enhanced by hypoxia in these patients. Methods: 34 OSA patients, median age $62 \mathrm{y}$, residing at $<600 \mathrm{~m}$ discontinued long-term CPAP therapy for 8 nights. They spent days 1 to 4 at low altitude and days 5 to 9 at high altitude in 2 mountain resorts (Davos Schatzalp, 1'850m, and Davos Jakobshorn, 2'590m), 2 days and nights each. They were randomized to undergo clinical evaluation and polysomnographies either during the last day in Zurich (490m) before ascent to altitude and during 4 days in Davos, or during 4 days in Davos and on the first day after their return to Zurich, respectively. Results: The table summarizes the results. Compared to Zurich, in the 1st night at Jakobshorn (2'590m), oxygen saturation and carbon dioxide tension were reduced while the apnea/hypopnea index was nearly doubled due to frequent central apnea in NREM sleep. Slow wave sleep was reduced and arousals were more prevalent. Heart rate and prevalence of premature beats were increased, blood pressure was elevated and patients had gained weight at altitude. Conclusions: These findings indicate that untreated OSA patients at altitude experience a further destabilization of their ventilation by hypoxic ventilatory stimulation causing hypocapnia and a reduced $\mathrm{CO} 2$ reserve. Consistent with ventilatory control theory, central apnea at altitude prevails mostly in NREM sleep. Hypoxia, pronounced oscillatory breathing, disturbed sleep and enhanced cardiovascular stress with water retention are all potentially serious adverse effects that occur even during a short-term sojourn at moderate altitude in untreated OSA patients. Therefore, effectiveness of CPAP and other treatment modalities should be evaluated in this setting.

Effect of altitude exposure in untreated OSA patients

\begin{tabular}{|c|c|c|}
\hline & Zurich, $490 \mathrm{~m}$ & Davos, 2’590m, $1^{\text {st }}$ night \\
\hline $\mathrm{SpO}_{2}(\%)$ & $94(93 ; 95)$ & $86(84 ; 89)^{*}$ \\
\hline $\mathrm{PtcCO}_{2}(\mathrm{mmHg})$ & $47(44 ; 50)$ & $37(34 ; 42)^{*}$ \\
\hline AHI total $(1 / \mathrm{h}$, total sleep time $)$ & $51(32 ; 74)$ & $30(62 ; 103)^{*}$ \\
\hline AHI obstructive (1/h, NREM) & $42(21 ; 62)$ & $31.1(3 ; 52)$ \\
\hline AHI central $(1 / \mathrm{h}, \mathrm{NREM}$ & $3(1 ; 10)$ & $54(31 ; 78)^{*}$ \\
\hline AHI obstructive (1/h, REM) & $36(21 ; 63)$ & $34(0 ; 65)$ \\
\hline AHI central (1/h, REM) & $3(0,2)$ & $10(1 ; 38)^{*}$ \\
\hline NREM stage $3+4(\%)$ & $14(8 ; 23)$ & $6(0 ; 12)^{*}$ \\
\hline REM (\%) & $13(9 ; 18)$ & $10(4 ; 16)^{*}$ \\
\hline Sleep efficiency (\%) & $86(80 ; 89)$ & $78(61 ; 84)^{*}$ \\
\hline Arousal index $(1 / \mathrm{h})$ & $36(25 ; 43)$ & $51(38 ; 66)^{*}$ \\
\hline Heart rate $(1 / \mathrm{min} \mathrm{TIB})$ & $57(55 ; 62)$ & $66(58 ; 72)^{*}$ \\
\hline Premature beats (1/h TIB) & $3(1 ; 11)$ & $7(2 ; 30)^{*}$ \\
\hline BP systolic $(\mathrm{mmHg}) \#$ & $130(120 ; 140)$ & $144(132 ; 160)^{*}$ \\
\hline BP diastolic (mmHg)\# & $85(80 ; 90)$ & $85(81 ; 90)$ \\
\hline Weight $(\mathrm{kg}) \#$ & $99(83 ; 111)$ & $100(85 ; 112)^{*}$ \\
\hline
\end{tabular}

$\mathrm{n}=34$, medians (quartiles) of polysomnographicdata. Variables marked with \# were measured in the following morning. $\mathrm{AHI}=$ apnea/hypopnea index, $\mathrm{SpO} 2=$ oxygen saturation, $\mathrm{PtcCO} 2=$ transcutaneous carbon dioxide tension, $\mathrm{NREM} / \mathrm{REM}=($ non)rapid eye movement sleep, $\mathrm{TIB}=$ time in bed, $\mathrm{BP}=$ blood pressure, ${ }^{*} \mathrm{p}<0.05$ vs. $490 \mathrm{~m}$.

7 Imaging the Homeostatic and Circadian
Contributions in PER2 Protein in Living Mice

T. Curie, V. Mongrain, S. Dorsaz, S. Maret, Y. Emmenegger, P. Franken

Center for Integrative Genomics, University of Lausanne, Switzerland

Sleep is regulated by two main processes: a homeostatic process that regulates sleep need and a circadian process that ensures that sleep occur at the appropriate time of day. Although the two processes interact to shape the distribution and quality of sleep and waking they are thought to act independently. Recent observations demonstrate, however, that these processes have more in common that was previously thought. Our published results show that circadian clock gene expression, that of Period 2 (Per2) in particular, increases during waking and decreases during sleep. These and other results suggest a non-circadian role for clock genes in sleep homeostasis. Here we investigate the dynamics of PER2 protein levels as a function of time-of-day and as a function of time-spent-awake (i.e., sleep deprivation) using C57BL/6J 
PER2::Luciferase knock-in mice. With the Xenogen 3-D imaging system, we could detect bioluminescence throughout the body, notably the brain (using a glass cylinder positioned onto the skull), and the liver and kidneys as verified using 3-D reconstitution software (Living 3-D image software). The luciferase substrate, luciferin, was infused constantly using osmotic minipumps either subcutaneously (for peripheral tissues) or ICV (for the brain). We observed diurnal changes in PER2 protein levels in the brain and the periphery. After $6 \mathrm{~h}$ sleep deprivation, we also observed an increase in bioluminescence from the brain and from the periphery, indicating an increase in PER2 protein consistent with the elevation of Per 2 mRNA we reported previously. The increase in PER2 was still important $2 \mathrm{~h}$ after the end of the sleep deprivation, while mRNA immediately decreased with recovery sleep. Preliminary western analyses in wild-type C57BL/6J mice seem to confirm these results. These results show, for the first time, that PER2 protein levels can be followed around the clock in the whole living mice and that PER2 protein is increased with sleep loss. We are currently developing a system to monitor PER2 expression in specific brain regions ( $\mathrm{SCN}$ and cerebral cortex) to more precisely dissect the circadian and homeostatic contributions to PER2.

\section{Poster Presentations}

\author{
8 \\ The Period Length of the Human Circadian \\ Clock Measured in Fibroblasts and in Vivo \\ Suggests Input-driven Plasticity \\ L. Pagani', C. Cajochen", D.J. Skene 3 , S.A. Brown", \\ A. Eckert \\ ${ }^{1}$ Neurobiology Laboratory for Brain Aging and Mental \\ Health, University of Basel, Psychiatric University Clinic \\ Basel, ${ }^{2}$ Center of Chronobiology, Psychiatric University \\ Clinic Basel, University of Basel, Switzerland, \\ ${ }^{3}$ Chronobiology Laboratory, School of Biological Sciences, \\ University of Surrey, UK, ${ }^{4}$ Institute of Pharmacology and \\ Toxicology, University of Zurich, Switzerland
}

Aims: Human behaviour is governed by the period length of a circadian clock in the suprachiasmatic nucleus of the brain hypothalamus. Measurement of this period requires extensive subject observation under controlled-light laboratory conditions. However the molecular mechanisms of the circadian clock are conserved in most cells of the body. In this study, we have for the first time directly compared human fibroblast period length with the free-running period length of human circadian behaviour measured in the same subjects in order to establish whether fibroblasts can be a good model for the study of the circadian rhythms. Methods: In vivo circadian period length from 10 sighted subjects has been measured in a Short Sleep Wake Cycle Constant
Routine Protocol. The in vivo period length from 8 blind subjects has been measured by weekly collection of urine and analyses of 6-sulphatoxymelatonin content for 3 weeks. From all the subjects participating to the study 2 skin punch biopsies have been taken and fibroblasts have been isolated and infected with an engineered lentiviral circadian reporter (mBmal-1::luc) to characterize the circadian rhythms in vitro by bioluminescence imaging. Measurements were conducted over a time period of 5 days under standard experimental conditions using Dulbecco's Modified Eagle's Medium containing 10\% foetal bovine serum. Results: A statistical significant correlation has been observed between fibroblast period length and in vivo period length measured in the same subjects, though fibroblast period values were on average slightly longer. The good correlation has been found both in the sighted as well as in the blind group. There is a statistical significant difference between circadian period length of the sighted group and of the blind group, probably reflecting the two different protocols used for the determination of in vivo period. However, the analyses of the in vitro circadian rhythms revealed that the period length of fibroblasts from the two groups of subjects is not different. Conclusions: Our data indicate that human "free-running" circadian period can be approximated by measurements in fibroblasts that can be considered as a good model for the study of the circadian rhythms. Moreover, manipulation of fibroblasts is not expensive or labor-intensive such as human in vivo protocols, fibroblasts are easy to maintain, accessible to molecular genetic tools and can be easily obtained from human subjects.

9

\section{Human Molecular Circadian Rhythms and Ageing}

L. Pagani', J. Izakovic ${ }^{2}$, C. Cajochen ${ }^{3}$, S.A. Brown ${ }^{4}$, A. Eckert

${ }^{1}$ Neurobiology Laboratory for Brain Aging and Mental
Health, University of Basel, Psychiatric University Clinic
Basel, ${ }^{2}$ Department of Dermatology, University Hospital
Basel, Basel, ${ }^{3}$ Center of Chronobiology, Psychiatric
University Clinic Basel, University of Basel, ${ }^{4}$ Institute of
Pharmacology and Toxicology, University of Zurich, Zurich,
Switzerland

Aims: In animals such as in human beings ageing leads to several pathologies, including circadian rhythms disturbances. Nevertheless, the origin of this phenomenon is unknown. To better understand the rebound of ageing on the circadian rhythms we characterized the period length of skin fibroblasts, an exploited in vitro model of peripheral oscillator, from 18 young and 18 elderly subjects. Methods: Fibroblasts were isolated from 2 skin punch biopsies and infected with an engineered lentiviral circadian reporter (mBmal-1::luc) to characterize the circadian rhythms in vitro by bioluminescence imaging. Measurements were conducted over a time period of 5 days under standard experimental conditions using Dulbecco's Modified Eagle's Medium containing 10\% foetal bovine serum (FBS) or 10\% of human serum. In addition, we evaluated data on the chronotype of the subjects by analysing 
the Munich Chronotype Questionnaire (MCTQ). Results: From the analyses of the MCTQ, the young subjects recruited for the study have an early chronotype compared to the subjects belonging to the older group; however no influence on the circadian period length of fibroblasts has been found upon the gender and the age in standard experimental conditions. To better understand the influence of age-related factors on the circadian rhythms, human fibroblasts have been measured in human serum containing medium. No difference in period length was found between treatment with FBS and "young" serum. In contrast, treatment with serum from older donors significantly reduced the circadian period length. To unmask the nature of the substance responsible for the influence of human serum on fibroblasts period length, sera have been heat-inactivated. Heat-inactivation of sera from older donors almost undid the reduction in the circadian period length suggesting that protein/s is/are responsible for the circadian disturbances observed in elderly. Conclusions: Data of this study reveal that there is no correlation between circadian period length and age at a molecular level. Thus apparently, the interplay between the molecular components of the skin fibroblasts oscillators does not change during ageing in general. However, the agerelated changes in circadian rhythms in vivo are possibly related to still unkown protein/s present in the serum.

Supported by grants fromEU-CLOCK fromE.U.\#LSHM-CT-2006-018741, from Désireé \& Niels Yde Fundation and from Fonds der Freiwilligen Akademischen Gesellschaft Basel.

\section{0}

\section{Melatonin and Peripheral Molecular Circadian Rhythms}

\author{
L. Pagani ${ }^{1}$, F. Meier'1, C. Cajochen ${ }^{2}$, A. Eckert ${ }^{1}$ \\ ${ }^{1}$ Neurobiology Laboratory, Psychiatric University Clinic \\ Basel, University of Basel, ${ }^{2}$ Center of Chronobiology, \\ Psychiatric University Clinic Basel, University of Basel, \\ Switzerland
}

Aims: Melatonin (Mel) is the principal substance synthesized and secreted by the pineal gland during the darkness. Despite Mel is one of the main markers of the circadian rhythms its physiological function is still unclear. To investigate the effects of Mel as a chronobiotic substance, we characterized the circadian rhythms of human peripheral oscillators in the presence of different concentrations of Mel. Methods: Human fibroblasts were isolated from 2 skin punch biopsies and infected with an engineered lentiviral circadian reporter (mBmal-1::luc) to characterize the circadian rhythms in vitro by bioluminescence imaging. The influence of Mel on the circadian rhythms was investigated in different experimental conditions. Results: The treatment of fibroblasts with Mel did not increase the synchronization of the cells. Cells that have been treated with a rather low concentration of Mel $(5 \mathrm{nmol} / \mathrm{ml})$ showed a dampening rate similar to cells treated with vehicle; instead fibroblasts treated with a rather high concentration of $\mathrm{Mel}(500 \mathrm{nmol} / \mathrm{ml})$ showed a higher dampening rate. To better understand the rule of Mel on the circadian rhythms, cells were measured with different concentrations of
Mel (from $5 \mathrm{nmol} / \mathrm{ml}$ up to $500 \mathrm{nmol} / \mathrm{ml}$ ). Low Mel concentration did not influence the circadian rhythms, whereas higher Mel concentrations reduced the circadian period length of peripheral oscillators. The influence of Mel on the circadian period length is present only after at least one day of pre-incubation of cells with Mel. To investigate the pathway through which Mel acts on the circadian rhythms, cells were treated with Mel and a Gi-inhibitor. The treatment with Gi-inhibitor alone did not shorten the period length compared to untreated cells, whereas treatment with Mel or Mel and Gi-inhibitor shortened the fibroblasts circadian period length of. Conclusions: From this study we can conclude that $\mathrm{Mel}$ is not able to synchronize peripheral oscillators, but it seems able to regulate the speedy of the period length. The effect of Mel on the length of the circadian period was stronger when cells were treated with Mel for at least 24 hours. Furthermore the effects of Mel on fibroblasts do not seem to be mediated via Gi-coupled proteins.

\section{1 \\ Sleep Disruption Aggravates Focal Cerebral Ischemia and Induces Expression of the Growth-inhibiting Gene Neurocan in the Rat}

\author{
B. Gao, E. Cam, H. Haeger, C. Zunzunegui, C.L. Bassetti \\ Dept. Neurology, University Hospital Zurich, Switzerland
}

Background/Aim: Sleewake disturbances (SWDs) are frequently observed in ischemic stroke patients and are linked with poorer function outcomes. The impact of sleep disruption on stroke pathophysiology is, however, unknown. The aim of this study was to investigate two sleep disruption procedures including sleep deprivation (SD) and sleep disturbance (SDis) on ischemia-induced brain damage and expression of neuroplasticity-related genes in a rat stroke model. Methods: Focal cerebral ischemia was induced by coagulating the distal middle cerebral artery (MCA) in Sprague Dawley rats $(n=44) .12 \mathrm{~h}$ after the ischemia surgery, SD was carried out by gentle handling. In the SD experiment, SD was carried out once and rats were sacrificed at the end of SD. In the SDis experiment, SD $12 \mathrm{~h}$ was carried out for 3 consecutive days in which rats were allowed to sleep for $12 \mathrm{~h}$ following SD. EEG was registered to evaluate vigilance states, infarct volumetry and TUNEL-positive cells were determined to assess brain damage, and the Taqman ${ }^{\circledR}$ PCR assay was for expression of several neuroplasticity-related genes, such as growth-promoting genes c-jun, gap43 and the growthinhibiting gene neurocan. The plasma corticosterone level was determined by Radio Immuno Assay. Results: Both SD12h and SDis significantly increased the infarct volume and number of tunnel-positive cells. Compared to the paired ischemia control groups, the infarct volume was increased by $41 \%$ in the SD $12 \mathrm{~h}$ $(94.1+16.9$ vs. $66.5+18.2 \mathrm{~mm} 3, \mathrm{p}=0.022)$ and $88 \%$ in the SDis group $(74.6+40.9$ vs. $39.7+43.4 \mathrm{~mm} 3, \mathrm{p}=0.023)$ respectively. The number of TUNEL-positive cells was increased by $137 \%$ in the SD $(46.8+15$ vs. $19.7+7.7 / \mathrm{mm} 2, \mathrm{p}<0.001)$ and $219 \%$ in the SDis group $(32.9+13.2$ vs. $10.3+2.5 / \mathrm{mm} 2, \mathrm{p}=0.002)$, respectively. SDis also significantly elevated the expression of neurocan $(14.3+0.4$ 
vs.6.2+0.1, $\mathrm{p}<0.001$ ), an axonal extension inhibitory molecule, in the injured hemisphere. Neither in the SD nor in the SDis experiment was the corticosterone level increased significantly. Conclusion: Sleep disruption aggravates ischemia-induced brain injury and alters expression of neuroplaticity-related genes. The impact of these changes on function recovery needs to be further investigated.

\section{2 \\ Ageing Leads to a Decrement of Cardiac Autonomic Control: Symbolic Analysis of Heart Rate Variability \\ A.U. Viola', S.L. Chellappa', E. Tobaldini², A. Porta², \\ K.R. Casali ${ }^{3}$, N. Montano ${ }^{2}$ \\ ${ }^{1}$ Centre for Chronobiology, University of Basel, \\ Switzerland, ${ }^{2}$ Department of Clinical Sciences, Internal \\ Medicine II, L. Sacco Hospital, University of Milan, Italy, \\ ${ }^{3}$ Department of Physiology, University of Rio Grande do \\ Sul, Brazil}

Aim: To test the hypothesis that symbolic indices of non-linear dynamics of heart rate variability (HRV) can track down agerelated effects and modulation by different sleep stages. Methods: Polysomnographic and electrocardiogram (ECG) recordings were performed in 12 young $(21.1 \pm 0.8 \mathrm{y})$ and 12 older $(64.9 \pm 1.9 \mathrm{y})$ healthy volunteers during one experimental night. Spectral analysis of HRV was performed as first step analysis. Symbolic analysis (SA) was done from ECG recordings and based on an automatic algorithm that constructed RR interval time series for each sleep stage (W, NREM, REM). SA and Shannon entropy (SE) assessments were then performed on time series of 4 epochs per sleep stage. SA encompasses conversion of time series into sequence of symbols and subsequent construction of series of patterns with three symbols, out of which four patterns can be identified: $0 \mathrm{~V}$ (no variation), $1 \mathrm{~V}$ (one variation), $2 \mathrm{LV}$ (two like variations) and 2UV (two unlike variations). The first is a marker of sympathetic activity, while the last refers to parasympathetic modulation. SE measures complexity of distribution patterns and reduced values indicate no relationship between subsequent patterns, which happens in aging and diseases. Results: During sleep, older subjects exhibit a fall in absolute HRV indices and a loss of sympathovagal sleestage dependant variation. Normalized $\mathrm{LF} /(\mathrm{LF}+\mathrm{HF})$ ratio remains sleestage dependant, albeit increased in older subjects. Sleep stages show significant differences in SA and SE measures in older subjects, with striking increase in $0 \mathrm{~V}$ pattern and decrease in 2UV pattern, together with reduced entropy rate. When considering sleep stages, these age-differences were significantly predominant during REM. Furthermore, comparisons between the two groups yielded a significant reduction in SE during REM, with increased $0 \mathrm{~V}$ and decreased $2 \mathrm{UV}$ patterns in older subjects. Conclusions: The fall of absolute HRV indices in older subjects indicates a decrement of the autonomic nervous system. Increase in $\mathrm{LF} /(\mathrm{LF}+\mathrm{HF})$ ratio during sleep implies that older subjects lose parasympathetic control on the autonomic balance. Non-linear dynamics and complexity of HRV undergo drastic age-changes, with older subjects exhibiting attenuated complexity and augmented non-linearity in the sympathetic domain, particularly during REM sleep. The main implication is that this sleep stage in older subjects may represent a physiological state risk for cardiovascular events.
13

\section{A Negative Relationship Between Sleep Duration And Intellectual Abilities from Childhood Through Adolescence: Findings from the Zurich Longitudinal Studies}

\section{O.G. Jenni, L. Molinari, J. Caflisch \\ Child Development Center, University Children's Hospital Zurich, Switzerland}

Introduction: A large body of adult research indicates that certain types of learning and cognitive processes depend on specific characteristics of sleep. However, these issues have only rarely been examined in children and adolescents. Methods: We studied the association between sleep duration and intellectual abilities in 493 children and adolescents of the Zurich Longitudinal Studies (born between 1974 and 1993, 352 term born children and 141 preterm born children). Structured face-tface interviews with parents were performed for various sleerelated habits: among other variables, bedtime, wake time, and daytime sleep duration were asked for the 3 months preceding the consultation. Mean sleep duration (mean standard deviation scores, SDS) from age 1 to 16 years was calculated from bedtime and wake time including day time sleep duration (= time in bed per 24 hours). Intellectual abilities were assessed using two different instruments: the German version of the WISC-R at ages 7, 9 and 14 years and the German AID at ages 11, 13 and 16 years. Results: A consistent negative correlation at all ages between mean SDS sleep duration and the intelligence quotient (IQ) was found. Both verbal and performance subscales contributed to the effect. Partial correlations (controlled for birth date, prematurity and SES) between mean sleep duration and IQ were between -0.172 and -0.544 . A hierarchical model including the covariates gender, prematurity, SES and birth date showed that IQ was significantly higher by an average of 3 IQ points per hour less sleep. While birth date was not related to IQ, prematurity and SES both showed the expected effect: former preterm children exhibited consistently lower intellectual abilities (mean 3 IQ points) than term born children and the average difference between the lowest and highest SES class was 20 IQ points. Conclusions: Individuals with a short sleep duration showed higher intellectual capacities than those with a long sleep duration. Thus, individual differences in sleep duration and individual variation in intellectual abilities may share common neurobiological underpinnings. 


\section{4}

\section{Quantification of the Periodicity of Inter Movement Intervals of Leg Movements}

\author{
C. Rummel, H. Gast, K. Schindler, J. Mathis \\ Dept. of Neurology, Inselspital Bern, Switzerland
}

Aims: Periodic leg movements in sleep (PLMS) are a frequent finding in polysomnography. Most patients with restless legs syndrome (RLS) display PLMS. However, since PLMS are also often recorded in healthy elderly subjects, the clinical significance of PLMS is still discussed controversially. Leg movements are seen concurrently with arousals in obstructive sleep apnoea (OSA) may also appear periodically. Quantitative assessment of the periodicity of LM/PLM as measured by inter movement intervals (IMI) is difficult. This is mainly due to influencing factors like sleep architecture and sleep stage, medication, inter and intra patient variability, the arbitrary amplitude and sequence criteria which tend to broaden the IMI distributions or make them even multi-modal. Methods: Here a statistical method is presented that enables eliminating such effects from the raw data before analysing the statistics of IMI. Rather than studying the absolute size of IMI (measured in seconds) we focus on the shape of their distribution (suitably normalized IMI). To this end we employ methods developed in Random Matrix Theory (RMT). Patients: The periodicity of leg movements (LM) of four patient groups (10 to 15 each) showing LM without PLMS (group 1), OSA without PLMS (group 2), PLMS and OSA (group 3) as well as PLMS without OSA (group 4) are compared. Results: The IMI of patients without PLMS (groups 1 and 2) and with PLMS (groups 3 and 4) are statistically different. In patients without PLMS the distribution of normalized IMI resembles closely the one of random events. In contrary IMI of PLMS patients show features of periodic systems (e.g. a pendulum) when studied in normalized manner. Conclusions: For quantifying PLMS periodicity proper normalization of the IMI is crucial. Without this procedure important features are hidden when grouping LM/PLM over whole nights or across patients. The clinical significance of PLMS might be eluded when properly separating random LM from LM that show features of periodic systems.

\section{5}

\section{Catechol-Methyltransferase (COMT) Val158Met Genotype Predicts Effects of Modafinil on Recovery Sleep EEG After Sleep Deprivation}

\author{
S. Bodenmann 1 , V. Bachmann 1,2, C. Stol/1, E. Geissler ${ }^{1}$, \\ K. Jaggi-Schwarz' ${ }^{1}$ H.P. Landolt ${ }^{1,2}$ \\ ${ }^{1}$ Institute of Pharmacology and Toxicology, University of \\ Zurich, Zurich, ${ }^{2}$ Zurich Center for Integrative Human \\ Physiology, University of Zurich, Zurich, Switzerland
}

Although the mode of action of modafinil remains to be debated, dopaminergic mechanisms related to dopamine re-uptake and dopamine D1 and D2 receptors contribute to the stimulant effects of the drug. Our laboratory recently demonstrated a role for catechol-methyltransferase (COMT), an important breakdown enzyme of cortical dopamine, in the efficacy of modafinil in healthy volunteers. Specifically, the Val158Met single nucleotide polymorphism of COMT, responsible for a 3 - 4 fold reduction in activity of this enzyme, predicts subjective and objective efficacy of modafinil after sleep deprivation. Homozygous carriers of the high-activity Val allele show better response to modafinil in alleviating impaired subjective well-being, sustained vigilant attention and executive functioning than individuals with low-activity Met/ Met genotype. We further investigated the interaction of modafinil with sleep wake-regulation in a double-blind, placebcontrolled study in healthy men (age: $23.4 \pm 0.5$ years). Two matched groups of $10 \mathrm{Val} / \mathrm{Val}$ and $12 \mathrm{Met} / \mathrm{Met}$ allele carriers completed 40 hours of prolonged wakefulness on two occasions separated by one week. Administration of $100 \mathrm{mg}$ modafinil or placebo was scheduled after 11 and 23 hours of wakefulness. Subjective sleepiness was assessed and the waking EEG recorded at 3-hour intervals. A baseline and recovery night in the sleep laboratory preceded and followed the sleep deprivation. Independent of COMT genotype, modafinil reduced subjective sleepiness and EEG theta activity (5-8 Hz) in the waking EEG, a suggested objective marker of sleep pressure during wakefulness. Sleep state analyses revealed no reliable effects of modafinil in recovery sleep. Nevertheless, in $\mathrm{Val} / \mathrm{Val}$ allele carriers, modafinil increased $2.75-6.25 \mathrm{~Hz}$ activity in nonREM sleep (stages 2-4) when compared to placebo. No differences between modafinil and placebo were observed in Met/ Met subjects. Based on these results, in combination with our earlier findings, we suggest that sleep deprived Val/Val allele carriers benefit more from modafinil than Met/Met allele carriers. Wakefulness after modafinil may be more intensive in these subjects when compared to placebo, and underlie the observed EEG changes in recovery sleep. Our data further indicate a dissociation between subjective and suggested EEG markers of sleep propensity in waking and sleep.

\section{6 \\ Cognition and High Density EEG in Sleep after Paramedian Thalamic Stroke}

\author{
R. Poryazova' ${ }^{1}$ R. Khatami ${ }^{1}$, E. Werth ${ }^{1}$, P. Brugger ${ }^{1}$, \\ R. Huber ${ }^{2}$, C.L. Bassetti ${ }^{1}$ \\ ${ }^{1}$ Department of Neurology, University Hospital Zurich, \\ University Children's Hospital Zurich, Zurich, Switzerland
}

Objectives: Functional recovery after stroke depends on the adaptive plasticity of the human brain. Sleep contributes essentially to brain plasticity and learning. The exact link between sleep, EEG and cognition in stroke recovery remains unclear. The aim of the study is to investigate the relationship between sleep and stroke recovery by: 1) comparing EEG power in the slow wave (SWA) and spindle frequency ranges (SFR) in the acute phase after stroke and 3 months later, 2) correlating these EEG parameters to clinical and behavioral changes during the recovery process.Patients and methods: Seven patients, two with bilateral paramedian thalamic stroke (PMTS) and five with uni- 
lateral PMTS and five matched controls underwent detailed neuropsychological examination, actigraphy, 24h-polysomnography (PSG) and high-density EEG (hd-EEG) during sleep. The patients were studied in the acute phase after stroke and 3 months later. Results: Patients performed worse in verbal fluency test, had longer total sleep time in PSG and higher percentage of day rest in actigraphy (acute and chronic). The patients with bilateral PMTS had more profound hypersomnia and impairment in behavioral tests. In hd-EEG both SWA and sleep SFR were recorded in typical locations without gross asymmetry. In comparison to healthy controls patients had significantly lower power in SWA in frontal and occipital regions after 3 months; power in SFR was lower although not statistically significant. After 3 months hypersomnia and behavioral tests improved, especially in the patients with bilateral PMTS. A significant decrease in the SWA power was found while power in SFR did not change. Conclusion: Behavioral and EEG changes were more profound after bilateral than after unilateral PMTS. Power in SWA after PMTS decreased after 3 months and was significantly lower than in controls while power in SFR was low initially and did not change. Improvement of cognition in bilateral PMTS was not accompanied by increase in SFR or SWA power.

The study is supported by the Zurich Center for Integrative Human Physiology (ZIHP).

\section{7}

\section{REM Sleep Behavior Disorder in Parkinson's Disease: A Questionnaire Based Study}

\author{
R. Poryazova, M. Oberholzer, F. Siclari, C.L. Bassetti \\ Department of Neurology, University Hospital Zurich, \\ Zurich, Switzerland
}

Objectives: Rapid eye movement sleep behavior disorder (RBD), reported in up to $50 \%$ of patients with Parkinson's disease (PD), is characterized by loss of normal muscle atonia during REM sleep which leads to increased phasic motor activity and allows dream enactment behavior. There are three previous large questionnaire and interview based studies addressing RBD in PD including 289, 200 and 231 patients respectively. The aim of the present study was to assess the frequency and the characteristics of RBD in patients with PD. Methods: A questionnaire including items on sleep quality, sleep disorders, PD characteristics and severity, was sent to the members of the national PD patients' organization in Switzerland. A 13-item validated questionnaire for RBD was included. A cut-off of five points is considered suggestive for RBD. Results: 420 questionnaires were received. Three patients had to be excluded for diagnoses other than idiopathic PD. 210/417 patients (50\%) had an RBD score $>5$. These patients reported concomitant sleep disorders like initial insomnia $(p=0.03)$, night-time awakenings $(p=0.003)$, apneas $(p=0.008)$, shortness of breath $(p=0.005)$, talking/crying in sleep $(p<0.001)$, cursing/violent behavior in sleep $(p<0.001)$, restless legs symptoms $(p<0.001)$, nightmares $(p<0.001)$ and hallucinations $(p<0.001)$ significantly more often then the rest of the population studied. They also had longer disease duration $(\mathrm{p}=0.04)$ and higher Epworth sleepiness score $(\mathrm{p}=0.019)$. There was a trend for lower score on activities of daily living $(\mathrm{p}=0.08)$. Conclusion: $\mathrm{RBD}$ in PD patients is associated with various sleep disorders, including insomnia, sleep disordered breathing, restless legs, nightmares and hallucinations, leading to higher arousability and sleep fragmentation. Patients with RBD were also sleepier than patients without RBD.

\section{8 \\ Sleepwalking in Patients with Parkinson's Disease}

R. Poryazova, M. Oberholzer, C.L. Bassetti

Department of Neurology, University Hospital Zurich, Zurich, Switzerland

Objectives: Sleepwalking (SW) corresponds to a complex sleeassociated behavior, which includes locomotion, mental confusion, and amnesia. SW is present in about $10 \%$ of children and $2-4 \%$ of adults, but only $0.6 \%$ of adults report SW "de novo". In a series of 165 consecutive patients with Parkinson's disease (PD) we found SW "de novo" in $6(3.6 \%)$. The aim of the present study was to assess the frequency and the characteristics of SW in patients with PD. Methods: A questionnaire including items on sleep quality, sleep disorders, in particular SW and REM sleep behavior disorder (RBD), PD characteristics and severity, was sent to the members of the national PD patients organization in Switzerland. Results: 420 questionnaires were received. Three patients had to be excluded for diagnoses other than idiopathic PD. 36/417 patients $(9 \%)$ reported adult SW, of them $22(5 \%$ of the population studied) had SW "de novo". Patients with SW had significantly longer disease duration $(p=0.035)$, they reported more often hallucinations $(p=0.004)$ and nightmares $(p=0.003)$, their dream content was more variable $(p=0.046)$ and they had higher scores, suggestive for RBD in a validated questionnaire $(\mathrm{p}=0.001)$. Patients with SW also had a trend for a higher Epworth sleepiness scale score $(\mathrm{p}=0.055)$. Conclusion: Our results suggest that SW in PD patients is more common then in the general populations. SW appears to be a late manifestation of PD. Most patients with SW also had questionnaire scores suggestive for RBD. The simultaneous occurrence of $\mathrm{SW}$ and RBD suggests a complex disturbance of arousal, locomotion and muscle tone during REM and NREM sleep in PD. 


\section{9 \\ Time Dependent Effects of Levodopa/ \\ Benserazide (LD/B) and Pramipexole (PPX) \\ on Periodic Leg Movements (PLM) in \\ Patients with Idiopathic Restless Legs Syndrome (RLS)}

\author{
D. Eggenberger ${ }^{1}$, C.L. Bassetti ${ }^{2}$, J. Mathis ${ }^{1}$ \\ ${ }^{1}$ University of Bern, Inselspital Bern, ${ }^{2}$ University Hospital \\ Zurich, Zurich, Switzerland
}

Objective: To compare time dependent treatment effects of the dopamine-agonist pramipexole (PPX; SIFROL ${ }^{\circledR}$ ) with a halfduration (time with a plasma concentration higher than $1 / 2$ Cmax; t1/2) of 8-12 hours with dual-release levodopa/benserazide (LD/B; MADOPAR DR $\left.{ }^{\circledR}\right)(\mathrm{t} 1 / 2=3-4$ hours $)$ on periodic limb movements (PLM) in patients with idiopathic restless legs syndrome (RLS). Methods: Post-hoc analysis of the PLM-indices of the first to eighth hour (PLM/h1-8) in bed at baseline and under treatment with PPX or LD/B obtained from 46 patients in the double-blind, randomized, Swiss multicenter, comparative crossover trial performed in 2006* (submitted for publication). Results: Under baseline conditions the PLM-index was significantly larger in the first four hours (PLM/h1-4) compared to the second half of the night $(\mathrm{PLM} / \mathrm{h} 5-8)(\mathrm{p}<0.001)$. Under treatment PPX and LD/B showed a statistically significant reduction of the PLM-indices in both halves of the night compared to baseline ( $p<0.001$ all-over). For both drugs the decrease of PLM/h1-4 was significantly larger compared to the decrease of PLM/h5-8 ( $p<0.001$ for PPX and $p$ $=0.027$ for LD/B): PPX reduced the median PLM/h1-4 from 35.8 to $9.0(\mathrm{p}<0.001)$ and the median PLM/h5-8 from 15.7 to 7.4 (p $<0.001)$, whereas LD/B decreased the median PLM/h1-4 from 32.2 to $11.0(\mathrm{p}<0.001)$ and the median PLM/h5-8 from 21.8 to $13.1(\mathrm{p}<0.001)$. Statistical analysis of the PLM-reduction under treatment demonstrated no significant difference between the two drugs, neither in the first four hours of bedtime $(p=0.207)$, nor in the second part of the night $(\mathrm{p}=0.831)$. Conclusion: Both drugs, LD/B and PPX, significantly reduce the PLM-index not only in the first four hours (PLM/h1-4), but also in the second part of the night (PLM/h5-8). The absence of any difference between the two drugs supports the value of PPX as an alternative to the standard medication LD/B in the treatment of RLS patients. Conversely, the equal efficacy of both drugs in the second part of the night underlines the positive effects of the dual-release formulation of

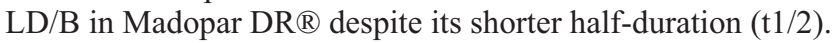

*The Swiss multicenter RLS Study 2006 was sponsored by BoehringerIngelheim

\section{0}

\section{Can Per3 Length Polymorphism Predict Sleep Duration in Older Individuals?}

\author{
F. Pugin', A.U. Viola', S.L. Chellappa', S. Archer², D.J. Dijk², \\ C. Cajochen ${ }^{1}$ \\ ${ }^{1}$ Centre for Chronobiology, Psychiatry University Clinics, \\ University of Basel, Switzerland ${ }^{2}$ Surrey Sleep Research \\ Centre, University of Surrey, UK
}

Aims: To investigate whether sleewake behaviour patterns measured by wrist actigraphy can differ between older subjects homozygous for the longer allele of the gene PERIOD3 (PER35/5) and heterozygous (PER34/5) individuals. Methods: Healthy volunteers were selected solely on the basis of their genotype, irrespective of diurnal preference or any other sleerelated characteristics. Approximately, 150 healthy older men and women (age 55-75) have been genotyped for the PER3 polymorphism in an ongoing study, thus comprising individuals who are homozygous for the longer (PER35/5) and for the shorter allele (PER34/4) as well as heterozygous individuals (PER34/5). In parallel, subjective assessment of sleep and chronotype was carried out using the Pittsburgh sleep quality index (PSQI), the Horne-Östberg and the Munich Chronotype Questionnaire, the Insomnia severity index and the Epworth sleepiness scale. So far, 23 healthy older subjects have completed sleep analysis by actigraphy and sleep diaries to characterize sleep timing during three consecutive weeks. In this preliminary dataset, we present data of 12 older participants, whose genotypes have already been characterized. Since only two PER34/4 subjects have completed this sleep analysis so far, they were not included in these results. Results: Preliminary results on 12 healthy older subject, six PER35/5 subjects and six PER34/5 subjects, indicate that there are no differences between the two genotypes with respect to age, gender, body mass index and to any of the sleepiness, sleep quality, insomnia index and chronotype questionnaires. On the other hand, the actiwatch analysis indicated highly significant differences between the two genotypes: PER35/5 subjects exhibited an earlier sleep time $(p=0.0001)$ and shorter sleep duration $(p=0.007)$ in comparison to PER34/5 subjects. Conclusions: These preliminary results point to an association between the length of the PER3 polymorphism and sleep duration in older subjects, which needs to be confirmed in further analysis. 
21

\section{Sleep and Waking EEG in Patients with Tetraplegia: Preliminary Findings}

\author{
K. Hefti ${ }^{1}$, H. Van Hedel ${ }^{2}$, V. Dietz ${ }^{2}$, H.P. Landolt ${ }^{1,3}$ \\ ${ }^{1}$ Institute of Pharmacology and Toxicology, University of \\ Zurich, ${ }^{2}$ Spinal Cord Injury Center, University Hospital \\ Balgrist, Zurich, ${ }^{3}$ Zurich Center for Integrative Human \\ Physiology, University of Zurich, Switzerland
}

Many patients with spinal cord injury (SCI) complain of disturbed nocturnal sleep and excessive daytime sleepiness. These symptoms could reflect changes in homeostatic and circadian processes of sleewake regulation. To better understand the underlying nature of sleewake disturbances in SCI, we are currently performing sleep and quantitative sleep and waking EEG studies in tetraplegic patients in comparison to healthy controls. To date, four patients ( 1 woman, 3 men; mean age: 43 years) with complete tetraplegia (Asia A) and four sex- and age-matched volunteers completed the study. Time between occurrence of injury and sleep studies equaled 3 months, 6 years, 19 years and 22 years. The study protocol includes all-night polysomnography in the hospital, 6 standardized waking EEG recordings at 3-hour intervals throughout the day following nocturnal sleep and sleepiness questionnaires. Saliva samples for melatonin quantification complete the current data collection. Our first results suggest similar sleep efficiency, sleep latency, REM sleep latency and sleep architecture in patients and controls. In the nonREM sleep EEG, however, the patients show higher power in delta (2.25-2.5 and 4.75-5.5 Hz range) and sigma (13.75-14.5 Hz band) frequencies. Also during wakefulness, the patients have generally higher absolute power values in the $0.75-4.75 \mathrm{~Hz}$ and $15.25-20.25 \mathrm{~Hz}$ ranges. By contrast, the evolution of relative power in distinct frequency bands across consecutive waking EEG recordings appears similar in both groups. Subjective ratings indicate elevated sleepiness in the patients in comparison to the controls, except at $4 \mathrm{pm}$. To our knowledge, this is the first quantitative sleep and waking EEG study in patients with complete tetraplegia. Preliminary findings indicate subtle differences in the sleep EEG between patients and controls, in particular in the frequency range of sleep spindles. Differences may also be present in the waking EEG. More patients are needed to corroborate the preliminary findings and to propose possible physiological changes contributing to the clinically observed sleewake symptoms in SCI.

\section{2}

\section{Vascular Dysregulation in Women Is Associated with Prolonged Sleep Onset Latency and Sleep Inertia: an Epidemiological Data Analysis}

\author{
K. Kräuchi', B. Gompper ${ }^{1}$, D. Anders' ${ }^{1}$, J. Flammer ${ }^{2}$, \\ S. Orgül ${ }^{2}$ \\ ${ }^{1}$ Thermophysiological Chronobiology, Centre for \\ Chronobiology, Psychiatric University Clinics, Basel, \\ ${ }^{2}$ University Eye Clinic, Basel, Switzerland
}

Introduction: The primary vascular dysregulation (PVD) syndrome is a functional disorder of blood flow regulation in otherwise healthy subjects (mostly women; leading symptoms: cold hands and feet). Women with PVD live not only with cooler extremities during the day but also exhibit a circadian phase delay of distal skin temperature rhythm with respect to their sleewake cycle (sleep timing) (Vollenweider et al., AJP 2008; Gompper et al., ESRS 2008). There is evidence from epidemiological, ambulatory and controlled laboratory studies of a close relationship between cold extremities in the evening and prolonged sleep onset latency (SOL) (Kräuchi, SMR 2008). Conversely, the time course of distal vasoconstriction after a sleep episode is correlated with the disappearance of sleep inertia (SI) (Kräuchi, JSR 2004). The aim of present epidemiological data analysis is to explore whether PVD, SOL, SI and sleep timing are interrelated. Methods: 145 women were recruited from participants of a larger survey in a random population sample of Basel-Stadt (Kräuchi et al., JSR 2008). Various questionnaires (questions about thermal discomfort, SI, SOL, sleep timing etc.) were mailed to the subjects. The degree of PVD was estimated from questionnaire-derived scores (feeling of cold hands and feet). Spearman rank correlations (Rsp) were calculated. Results: The degree of PVD was positively correlated with weekly means of SOL $(\mathrm{Rsp}=0.265$, $\mathrm{p}=0.0022)$, SI $(\mathrm{Rsp}=0.245, \mathrm{p}=0.0061)$ and sleep timing [(lights on - lights off $/ 2 ; \mathrm{Rsp}=0.234, \mathrm{p}<0.01]$. SOL correlated with SI $(\mathrm{Rsp}=229, \mathrm{p}=0.011)$, and sleep timing was weakly correlated with SI $(R s p=0.170 ; p=0.07)$, but not with $\operatorname{SOL}(R s p=0.086 ; p=0.35)$. Conclusions: These findings are in accordance with the hypothesis that prolonged SOL and SI are associated with delayed distal vasodilatation before and delayed distal vasoconstriction after sleep, respectively, leading to a phase delay of the thermoregulatory system with respect to sleep timing (different internal phase of entrainment). Different external phase of entrainment (different phase angle between sleep timing and solar-cycle) seems to be less crucial for SOL and SI.

Research supported by the Schwickert-Stiftung and SNF Grant \#3200B0116504 to K.K. 
23

Prevalence, Severity, Clinical Relevance, Pathophysiology and Course of the Restless Legs Syndrome in Pregnancy: A Prospective, Systematic Study

\author{
A. Hübner ${ }^{1}$, K. Krafft ${ }^{2}$, E. Werth ${ }^{1}$, S. Gadient ${ }^{1}$, \\ R. Zimmermann ${ }^{2}$, C.L. Bassetti ${ }^{1}$ \\ ${ }^{1}$ Department of Neurology, University Hospital Zurich, \\ ${ }^{2}$ Department of Gynecology, University Hospital Zurich, \\ Switzerland
}

Introduction: Three retrospective studies [1-3] suggested a high frequency $(11-26 \%)$ of restless legs syndrome (RLS) in pregnancy. None of these studies used standardized methods for assessment of RLS and sleep. Characteristics and determinants of RLS during pregnancy are poorly known. Methods: Women during (from the 2 nd trimester, $1 \mathrm{x} /$ month) and after pregnancy (8 weeks post-partum) are prospectively studied. Assessment includes 1) interview about RLS-symptoms and sleep habits/ disturbances: 2) standardized questionnaires (incl. the international RLS-scale (IRLSS), Epworth sleepiness scale (ESS), and Pittsburgh Sleep Quality Questionnaire (PSQI)); 3) blood tests (incl. hemoglobin, C- reactive protein and ferritin), 4) leg actigraphy (3rd trimester and post-partum). Results: So far 308 women were included. RLS was diagnosed in 28 women (9.1\%). $28 \%$ of them had a positive family history for RLS, $60 \%$ reported onset of RLS-symptoms before the 20th week, $60 \%$ had RLS-symptoms daily, $60 \%$ had an IRLSS $>20$, and $85 \%$ had a PSQI $>5$. Anemia (defined as $\mathrm{Hb}<11 \mathrm{~g} / \mathrm{dl}$ in pregnant women) was found in $10 \%$ of affected and unaffected women. Ferritin levels $<50$ were found in $88 \%$ of women. Women with (pRLS) and without RLS (nRLS) had similar values in hemoglobin CRP, and ferritin (mean $\mathrm{Hb} 11.8$ $\mathrm{mg} / \mathrm{dl}$ in $\mathrm{pRLS}, 12 \mathrm{mg} / \mathrm{dl}$ in nRLS; Ferritin $19.1 \mu \mathrm{g} / \mathrm{l}$ in pRLS, 13.3 in nRLS). Conclusion: Preliminary results of this ongoing study suggest that RLS in pregnancy: 1) is present in $10 \%$ of women; 2) frequently appears early in pregnancy; 3) is often severe/ frequent; 4) may not be related (only) to anemia/low ferritin levels; 5 ) has a significant impact on sleep quality.

1. Manconi M.,Govoni V., De Vito A., Economou N.T., Cesnik E., Casetta I., Mollica G., Ferini-Strambi L., Granieri E., Restless legs syndrome in pregnancy, Neurology 63/2004

2. Goodman J., Brodie C., Ayida G.A., Restless leg Syndrome in pregnancy, BMJ, Oct. 1988, Vol. 297;1101-1102

3. Ekbom K.A., Restless Legs, Acta Scand. Med. 1945;158:1-123

\section{4 \\ Slow Wave Sleep and Local Grey Matter Volumes}

\section{A. Buchmann, S. Kurth, M. Ringli, O.G. Jenni, R. Huber \\ University Children's Hospital Zurich, Switzerland}

Aims: Slow-wave activity (SWA; power $<4.5 \mathrm{~Hz}$ ) during nonREM sleep is a well established electrophysiological marker of sleep intensity. Previous research has shown a dramatic decrease of SWA in the sleep EEG during adolescence [1, 2], which seems to be paralleled by a decline in synaptic density [3]. Another anatomical correlate of development, the increase and following reduction of grey matter volumes during adolescence [4], can be assessed in vivo on MRI images. Based on the hypothesis that sleep actively supports developmental processes in the young brain, we looked for grey matter correlates of slow wave sleep (SWS, i.e. sleep with high SWA) duration in the brain. Methods: In a sample of 14 children (6 boys; age range 9-13 years, mean $=10.5 \mathrm{y}, \mathrm{SD}=1.2 \mathrm{y}$ ) we correlated local grey matter volumes with the percentage of SWS (mean of two nights). To achieve this, we used T1-weighted MRI images and segmented them in a fully automatic way using the SPM5 package (http:// www.fil.ion.ucl.ac.uk/spm). Grey matter segments were then smoothed with a $12 \mathrm{~mm}$ Gaussian kernel and compared voxel by voxel using voxel-based morphometry [4]. We calculated wholebrain and voxel-wise multiple regressions accounting for sex, age and whole-brain grey matter volumes. Results: Whole brain grey matter volumes (in our sample $891 \pm 19 \mathrm{ccm}$ ) did not correlate significantly with percentage time in slow wave sleep (Beta $=-.085$; $\mathrm{p}=.845)$. However, we found areas of grey matter correlating positively (Beta up to $.98 ; \mathrm{p}<.001$ uncorrected) with the duration of slow wave sleep, namely in anterior and posterior cinguli, in the medial temporal gyrus, lateral parietal lobe and in the ventromedial and dorsolateral prefrontal cortex. No significant negative correlations were found. Conclusions: Our preliminary anatomical data provide first evidence for a relationship between SWS duration and local grey matter volumes in regions of the brain, which are highly plastic during adolescence. Ongoing work will show if SWS-associated regions shift during adolescence.

\section{References}

1. Jenni O.G. \& Carskadon M.A., Sleep 27, 774-783, 2004.

2. Jenni O.G. et al., Sleep 28, 1446-1454, 2005.

3. Feinberg I, Journal of Psychiatry Research 17(4), 319-334, 1982.

4. Giedd J N et al., Nature Neuroscience 2, 861-863, 1999.

5. Ashburner J \& Friston K J, Neuroimage 11, 805-821, 2000.

\section{5}

\section{Behaviourally Induced Insufficient Sleep Syndrome and Its Borderland}

\author{
E. Werth, N. Michael, C.R. Baumann, C.L. Bassetti \\ Department of Neurology, University Hospital Zurich, \\ Switzerland
}

Introduction: Behaviourally induced insufficient sleep syndrome (BIISS) occurs when an individual chonically fails to obtain the amount of sleep required to maintain normal levels of alertness and wakefulness. Method: This study presents the results of the post hoc evaluation (Clinical evaluation, Sleep questionnaire (SQ), actigraphy, PSG, MSLT, MWT, HLA typing, hypocretin) of 47 consecutive patients who received the diagnosis of BIISS in our Center of Sleep Disorders. Results: Mean age of the BIISS patient was $40 \pm 12$ years (mean \pm SD). Only $30 \%$ were females. Patients mostly complain symptoms of hypersomnia with exces- 
sive daytime sleepiness, however, many individuals reported other symptoms as sleep attacks without general daytime sleepiness, fatigue, sleep drunkenness, concentration and attention deficits or cognitive impairment. Mean ESS was 14.1 \pm 3.6 . Time in bed (TIB) estimation based on the SQ revealed TIB of $7: 10 \mathrm{~h} \pm 1: 03 \mathrm{~h}$ during weekdays and $8: 29 \mathrm{~h} \pm 1: 16 \mathrm{~h}$ on weekend. TIB estimation based on actigraphy recordings revealed significantly shorter TIB on weekdays and on weekends (weekday: $6: 25 \mathrm{~h} \pm 0: 57 \mathrm{~h}$, weekend: $7: 56 \mathrm{~h} \pm 1: 13 \mathrm{~h}$ ) compared to TIB taken from the SQ. In this population the PSG recording revealed short sleep latency $8.4 \pm 7.9$ minutes and high sleep efficiency $(91.5 \pm 16.7 \%)$. Mean sleep latency of MSLT was 5.5 \pm 3.3 minutes. Sleep onset REM (SOREM) episodes with 2 and more SOREM were present in 8 patients. Mean sleep latency of MWT was very variable. A clear reduced ability to maintain wakefulness (sleep latency $<12 \mathrm{~min}$ ) was present in $34 \%$ of patients. Conclusion: The results of this case series indicate that there are a noticeable large number of patients who were not aware that their sleep duration was insufficient and that there is a substantial clinical overlap between BIISS, narcolepsy without cataplexy and idiopatic hypersomnia without long sleep. A positive response to increased sleep time is diagnostic of BIISS and an important feature to differentiate between these three entities.

\section{6 \\ Stability of Spectral Characteristics in the Sleep EEG During Early Adolescent Development: Preliminary Results}
L. Tarokh1,2, M. A. Carskadon 1,3, P. Achermann ${ }^{4,5}$
${ }^{1}$ Bradley Hospital Sleep Research Laboratory, Providence, R.I., ${ }^{2}$ Center for Alcohol and Addiction Studies, Brown University, Providence, RI, ${ }^{3}$ Department of Psychiatry and Human Behavior Warren Alpert Medical School at Brown University, Providence, RI, USA, ${ }^{4}$ Institute of
Pharmacology and Toxicology, University of Zurich, Zurich,
5Zurich Center for Integrative Human Physiology,
University of Zurich, Zurich, Switzerland

The waking and sleep EEG spectrum in adults is highly stable across recording sessions separated in time by several weeks. This phenomenon has not been studied in children and adolescents in whom brain development may affect the sleep EEG. The present longitudinal study examined whether the sleep EEG spectrum remains stable during the transition from childhood $(9 / 10$ years old) to early adolescence (11 - 13 years old). Standard sleep recordings were performed in ten healthy ( 3 girls) children on two consecutive nights when participants were 9/10 years old (time 1) and again $2-3$ years later (time 2 ; mean $=2.32$ years, $\mathrm{SD}=$ 0.28). EEG signals (C3/A2) were Fourier transformed (Hanning window, average of six 5-s epochs) and artifacts rejected using a semi-automated procedure; data were averaged across the night independent of vigilance states. Spectral power from 0.6 to 16 $\mathrm{Hz}$ (in $0.2 \mathrm{~Hz}$ bins) was normalized and $\log$ transformed before classification by hierarchical cluster analysis. At both times, the cluster analysis correctly paired consecutive recording nights for every participant. When all 40 nights were entered into the analysis, all four nights from each participant were correctly classified except for one participant, for whom the two nights at time 1 and time 2 were paired but not all 4 nights, possibly due to a 0.4 $\mathrm{Hz}$ increase in the spindle frequency peak at time 2. Our results indicate that - in spite of neurdevelopmental changes - trait-like characteristics are preserved in $90 \%$ of our sample across this developmental phase. Future analyses will include a larger sample size and examine NREM and REM sleep separately.

\section{7 \\ Sleep Patterns and Cognitive Development of Children: Is There a Common Link?}

\author{
A. Geiger ${ }^{1}$, P. Achermann ${ }^{2}$, O. G. Jenni ${ }^{1}$ \\ ${ }^{1}$ University Children's Hospital Zurich, Child Development \\ Center, Zurich Center for Integrative Human Physiology \\ (ZIHP), ${ }^{2}$ University of Zurich, Institute of Pharmacology \\ and Toxicology, Zurich Center for Integrative Human \\ Physiology (ZIHP), Zurich, Switzerland
}

Sleep and cognition are commonly linked - in terms of minimal sleep duration that is assumed for adequate cognitive functioning and in terms of developmental relevance. But even though a role for sleep in learning processes could be demonstrated in studies [1], the association between sleep and cognition on the trait-level is far less clear. Furthermore, most research about the relationship between sleep and cognition focuses on clinical populations or on the effect of disturbed sleep on cognitive performance. To specify this association in healthy children, 60 children aged 8-11 years were comprehensively characterized on cognitive (German version of the WISC IV) and attentional dimensions (alertness and go/nogo paradigm). Additionally, several sleep behavior parameters for the same children were assessed. Questionnaires, fourteen days of actigraphy recording and sleep diaries were used to specify sleep behavior parameters such as daytime sleepiness, sleep duration, and chronotype. In a correlative analysis, a negative correlation of $-.29(\mathrm{p}<.05)$ between full scale IQ scores and sleep duration (according to questionnaires filled out by parents) could be demonstrated with the main component being fluid intelligence $(r=-.35, p<.05$, partial correlation controlled for age and socioeconomic status). Thus, the shorter the sleep duration on free days, the higher the scores of cognitive performance. Attentional parameters did neither correlate with any of the cognitive variables nor with sleep behavior parameters. Based on the correlation analysis, regression analyses were conducted. We conclude that, children displaying higher cognitive efficiency during daytime (as reflected by higher IQ scores) seem to display higher night-time sleep efficiency (as reflected by shorter sleep duration). In light of the neural efficiency hypothesis [2], the current results argue for an upgrading of the original theory - referring not only to daytime but also night-time efficient behavior. After all, sleep as well as cognition can both be considered as substrates of neuronal processing and thus should indeed have some common properties.

1. Walker MP, Stickgold R: Sleep, memory, and plasticity. Annu Rev Psychol 2006, 57:139-166. 
2. Haier RJ, Siegel EV: Cortical glucose metabolism rate correlates of abstract reasoning and attention studied with positron emission tomography. Intelligence 1988, 12 (2): 199-217.

\section{8 \\ Source Localization of Slow Oscillations in the Nonrem Sleep EEG: Effect of Sleep Deprivation}

\section{A. Bersagliere, P. Achermann \\ Institute of Pharmacology and Toxicology, University of Zurich, Zurich, Switzerland}

Aims: Slow waves are the most prominent feature of nonREM sleep and are visible at the level of the scalp EEG as large amplitude fluctuations at frequencies ranging from slow $(<1 \mathrm{~Hz})$ to delta (1-4 Hz) activity [1]. At the cellular level they are characterized by slow membrane potential fluctuations of cortical neurons consisting of depolarized up states and hyperpolarized down states alternating synchronously over vast cortical territories with a frequency below $1 \mathrm{~Hz}$ [2]. Aim of the present analysis was to investigate changes in the activation of brain structures during slow oscillations following sleep deprivation. Methods: EEG recordings during baseline sleep and recovery sleep after 40 hours of wakefulness were analyzed ( 27 channels, 7 healthy young men, slow wave sleep (stages 3 and 4) of the first nonREM sleep episode). Slow oscillations were detected based on the Hilbert transformed EEG of derivation $\mathrm{Cz}$ (linked mastoids; band-pass filter 0.5-4.5 Hz; peak-tpeak amplitude $>75 \mu \mathrm{V}$ ). Source localization using the software LORETA [3] was performed for the downstates (short time interval around the negative peak). Results: Preliminary analysis revealed significant clusters of increased activation after sleep deprivation in the right frontal lobe and decreased activation in both parietal lobes, left limbic and temporal lobes. Conclusions: Further analyses are needed to investigate the contribution of the ustates.

1. Massimini M, Huber R, Ferrarelli F, Hill S, Tononi G: The sleep slow oscillation as a traveling wave. J Neurosci, 2004, 24: 6862-70.

2. Steriade M, Nunez A, Amzica F: A novel slow $(<1 \mathrm{~Hz})$ oscillation of neocortical neurons in vivo: depolarizing and hyperpolarizing components. J Neurosci, 1993, 13: 3252-65.

3. Pascual-Marqui RD, Michel CM, Lehmann D: Low resolution electromagnetic tomography: a new method for localizing electrical activity in the brain. International Journal of Psychophysiology 1994, 18:49-65.

Supported by SNSF grant 320000-112674.
29

\section{The Chronophysiological Dawn of Female Adulthood}

\author{
S. Frey ${ }^{1}$, S. Balu1 , S. Greusing ${ }^{1}$, N. Rothen ${ }^{2}$, C. Cajochen ${ }^{1}$ \\ ${ }^{1}$ Chronobiology, Psychiatric University Clinics, Basel, \\ ${ }^{2}$ Institute of Psychology, University of Bern, Bern, \\ Switzerland
}

Most parents experience their children's puberty as a dramatic change in their family life. This is not surprising considering the dynamics of the physical and psychosocial maturation period, which occur during adolescence. Although an age- and sexdependent turn in chronotype has been shown during adolescence chronological age may not give an accurate explanation for the physical and behavioural dynamics that occur before the transition from adolescence to adulthood. Hence, the aim of our study was to assess whether there is a relationship between puberty and the changes in sleep phase preferences during female maturation and adulthood. Our study is based on a cross-sectional survey among 906 females aged 5 to 29 years. Habitual sleep timing on weekdays and free days were investigated along with the date of birth and the year of the occurrence of menarche. The distance measured in years from the actual age to menarche onset was taken as a biological variable that may affect the change in sleep timing preference. To account for changes of sleep phase preferences the difference between the midpoints of sleep on free days and on weekdays was calculated which has also been aptly described as "social jetlag". Sleep phase preference was then subjected to a one-way ANOVA with the factor "distance to menarche". Results show that in contrast to prepubertal children, female adolescents exhibit a striking progression in delaying their sleep phase preference until 5 years after menarche. Thereafter, the mid-sleep difference switches to advancing. Results disclosed a main effect of the factor "distance to menarche" $(\mathrm{p}<0.0001)$. Post-hoc analysis showed that mid-sleep difference at -7 years to menarche and $9.5,11.5$, and 13.5 years after menarche are significantly different from the peak in mid-sleep difference at 5 years after menarche $(\mathrm{p}<0.0001)$. Our data provide evidence that the downsizing of social jetlag 5 years after menarche is a step towards "adultlike" behaviour as the ability to approach more aligned sleep/ wake cycles during weekdays and weekends emerges. Hence, we conclude that the distance to menarche may serve as an individual biological marker for the beginning of female adulthood.

Research supported by the Swiss National Science Foundation and the Daimler-Benz Foundation 


\section{0}

\section{Dynamics of Sleep Homeostasis: A Trait-like Feature?}

T. Rusterholz ${ }^{1,2}$, H.P.A. Van Dongen ${ }^{3}$, P. Achermann $n^{1,2,4}$

${ }^{1}$ Institute of Pharmacology and Toxicology, University of

Zurich, Zurich, ${ }^{2}$ Neuroscience Center Zurich, University and ETH Zurich, Zurich, Switzerland, ${ }^{3}$ Sleep and

Performance Research Center, Washington State

University, Spokane, Wash., USA, 4Zurich Center for

Integrative Human Physiology, University of Zurich, Zurich,

Switzerland

The homeostatic Process S reflects the prior history of sleep and wakefulness and is modeled by a saturating exponential function during waking and an exponential decline during sleep. EEG slow-wave activity (SWA) is a marker of nonREM sleep intensity and serves as an indicator of sleep homeostasis. There is considerable inter-individual variation in responses to sleep deprivation. The aim of the present study was to investigate whether the dynamics of sleep homeostasis (i.e. the time constants of Process S) are trait-like. EEG data of healthy young adults who underwent a laboratory protocol with repeated sleep deprivation was used to investigate this. Each subject's data consisted of EEG recordings from eight sleep periods (12 h time in bed, 22:00-10:00), which were interspersed with three $36 \mathrm{~h}$ periods of sustained wakefulness [1]. Empirical mean SWA per nonREM sleep episode at episode midpoint served as input for least-squares model parameter estimation. Time constants were constrained to a physiological range derived from average data. Parameters were estimated for 6 different pairings of the recording nights. Random-effects ANOVA was used to investigate the intra- and inter-individual variation in the estimates of the time constants. The standard deviation across subjects for the time constant of buildup was $4.9 \mathrm{~h}$, and the interindividual differences explained $59 \%$ of the variance. The standard deviation across subjects for the time constant of the decline was $0.3 \mathrm{~h}$, and inter-individual differences explained only $26 \%$ of the variance. Thus, there was substantial inter-individual variability in the dynamics of Process S during wakefulness but not during sleep. It remains to be determined to what extent the inter-individual differences during wakefulness are a byproduct of the model fitting procedure, or a result of systematic inter-individual differences in waking activities, or evidence of a trait-like feature.

1. Tucker AM, Dinges DF, Van Dongen HPA: Trait interindividual differences in the sleep physiology of healthy young adults. J. Sleep Res. 2007, 16:170 180 .

Supported by SNSF grant 320000-112674 and NIH grants HL70154 and RR00040.

\section{1 \\ Local Increase of Sleep SWA After Visuomotor Learning in Children}

\author{
M. Ringli, S. Kurth, A. Geiger, O.G. Jenni, R. Huber \\ University Children's Hospital Zurich, Zurich, Switzerland
}

Introduction: Slow wave activity (SWA) during NREM sleep is homeostatically regulated and was suggested to depend on synaptic changes during wakefulness [1]. In adults it was shown, that a visuomotor learning task involving specific brain regions resulted in a local increase of SWA during subsequent sleep [2]. Knowing that the brain undergoes dramatic changes during development $[3,4]$, we wondered if the same learning task would have similar effects on SWA in immature children. Methods: All-night high density EEG was recorded in twelve prepubertal children (mean age 10.9 \pm 1.3 years, right handed) using Geodesics Sensor Nets composed of 128 electrodes in two separate nights. The EEG recordings were sleep staged, subjected to semi-automatic artifact removal and processed using power spectral analysis. We calculated for every electrode power in the SWA frequency range (1-4.5 $\mathrm{Hz}$ ) for the first 30 minutes of NREM sleep for each night. Before sleep subjects completed with their right hand either a visuomotor learning task or a motor control condition. Results: Our children successfully learned the task. During subsequent sleep, we found a highly reproducible pattern of SWA topography for the two conditions. However, a contrast revealed a significant local increase of SWA over left central $(8.3 \pm 2.5 \% ; \mathrm{p}<0.05)$ and occipital regions $(8.6 \pm 3.3 \% ; \mathrm{p}<0.05)$ after the learning condition. Discussion: As observed in adults a preliminary analysis revealed local changes in SWA triggered by the learning condition in children. Other than in adults, where higher-order brain regions in the parietal cortex were found to be involved in the underlying learning processes, in children significant increases were observed over the left motor and visual regions. Based on evidence that higher-order brain regions mature later during development [3], our findings may suggest that in children lower-order brain regions seem to execute visuomotor function.

1. Tononi G, Cirelli C: Sleep and synaptic homeostasis: a hypothesis. Brain Res Bull 2003; 62: 143-50.

2. Huber R, Ghilardi MF, Massimini M, Tononi G: Local sleep and learning. Nature 2004; 430: 78-81.

3. Gogtay N, et al.: Dynamic mapping of human cortical development during childhood through early adulthood. Proc Natl Acad Sci U S A 2004; 101: 8174-9.

4. Sowell ER, et al.: Longitudinal mapping of cortical thickness and brain growth in normal children. J Neurosci 2004; 24: 8223-31. 


\section{2}

\section{Age-related Changes in Response to Sleep Deprivation and Caffeine}

\author{
R. Wehrle1, J.V. Retey 1 , M. Adam¹, H.P. Landolt ${ }^{1,2}$ \\ ${ }^{1}$ Institute of Pharmacology \& Toxicology, University of \\ Zurich, ${ }^{2}$ Center for Integrative Human Physiology, \\ University of Zurich, Switzerland
}

Aim: Recent evidence suggests that healthy older individuals are less vulnerable to performance impairment after sleep deprivation than young individuals. Some of the age-related differences may be reminiscent of the response in young people to the adenosine receptor antagonist caffeine. To study possible adenosinergic mechanisms contributing to age-related changes in sleewake regulation, we examined the effects of sleep deprivation and caffeine on sleepiness, daytime functions and EEG variables in young subjects of high and low self-rated caffeine sensitivity and healthy older men. Methods: Twelve young caffeine-sensitive $(23.7 \pm 3.3$ years $), 10$ young caffeine-insensitive $(25.0 \pm$ 2.1 years $)$, and 10 older volunteers $(66.1 \pm 3.3$ years $)$ were studied. All participants completed two 40 -hour periods of prolonged wakefulness in subsequent weeks preceded by two 8-hour nights in the sleep laboratory. In both conditions, two capsules were administered after 11 and 23 hours waking, containing either $2 \mathrm{x}$ $200 \mathrm{mg}$ caffeine or placebo in randomized, double-blind, crossover fashion. Subjective sleepiness (Stanford Sleepiness Scale), sustained vigilant attention (PVT), and waking EEG (eyes open, eyes closed) were obtained at 3-hour intervals throughout prolonged wakefulness. Significant effects of sleep deprivation and caffeine were identified with mixed-model ANOVAs. Results: Sleep deprivation impaired subjective sleepiness and PVT performance in all groups, with the sensitive young participants being most affected. The pharmacokinetics of caffeine in saliva did not differ among the groups. Notwithstanding, caffeine most potently improved sleepiness, and PVT measures following sleep loss in the young sensitive group, whereas insensitive and older subjects showed little effects. These differences among the groups were not paralleled by the effects of sleep deprivation and caffeine on delta and theta power in the waking EEG. Conclusions: Sleep deprivation and caffeine have less effect on subjective sleepiness and objective performance in older subjects than in young caffeine sensitive subjects. These differences may reflect age-related changes in adenosine and adenosine receptors, which are well established in animals and humans. A dissociation among sleepiness, performance, and putative EEG measures of alertness during prolonged wakefulness is suggested.

Research supported by Swiss National Science Foundation and EU MarieCurie grant MCRTN-CT-2004-512362.
33

\section{Sleep Dependent Performance Improvement in Children}

\author{
S. Kurth, M. Ringli, A. Geiger, O.G. Jenni, R. Huber \\ Children's Hospital Zurich, Switzerland
}

In adults, numerous reports show sleep dependent performance improvement on various tasks [1]. For example, subjects trained on a computer-based visumotor rotation task showed improved test performance after sleep [2]. We investigated a simplified version of this task in 16 children $(10.4 \pm 1.2 \mathrm{y}, 8$ males) and 8 adolescents/adults $(20.3 \pm 3.9 y, 3$ males $)$. In this task righthanded subjects using a computer mouse had to perform out and back movements towards a target as accurately and fast as possible. Unbeknown to the subjects they had to overcome a computer imposed rotation and trying to adapt their movements to hit the targets. A 25-min training session followed by a first test took place in the evening before sleep. Subjects were retested on the task next morning. We compared learning performance of the subjects during the training session, as well as the changes in test performance before and after sleep. Performance was assessed by the directional error (degrees of accuracy), the angle between the target and actual movement direction. At the end of the training session, similar levels of directional error were reached in children (25.5 degrees) and adolescents/adults (21.8 degrees). However, adolescents/adults reduced their movement variance across the training sessions (i.e. the precision of hitting targets) to a larger extent than children did (adolescents/adults, -24.5 degrees children -13.7 degrees, $\mathrm{p}<0.05)$. After sleep, both children and adolescents/adults showed improved task performance. The improvement was larger in adults $(+35.2 \pm 13.8 \%)$ than in children $(+10.8 \pm 4.7 \%, \mathrm{p}<0.05)$. We found no correlations between the after sleep performance improvement and sleep variables (sleep efficiency, NREM sleep, REM sleep). A possible explanation for the reduced sleep dependent task performance improvement in children might be their larger movement variance during the training session, i.e reduced movement accuracy, and/or a not yet fully matured visumotor integration system.

1. Born J, Rasch B, Gais S, Sleep to remember. Neuroscientist 2006; 12:410-424.

2. Huber R, Ghilardi MF, Massimini M, Tononi G, Local sleep and learning. Nature 2004; 430: 78-81.

\section{4 \\ Sleep Regulation in Mice with Adenosine Metabolism Deficiency}

S. Palchykova, A. Gerling, R. Winsky-Sommerer, I. Tobler

Institute of Pharmacology and Toxicology, University of Zurich, Switzerland

Introduction: It is suggested that the function of sleep is associated with energy metabolism. Adenosine is a neuromodulator implicated in sleep promotion and regulation due to its ability 
to decrease neuronal activity and due to a direct link of adenosine to the energy metabolism in the cell. It has been shown by microdialysis in cats and rats that adenosine increases during prolonged wakefulness. Moreover, adenosine, its analogs and adenosine receptor agonists induce sleep, while adenosine receptor antagonists induce waking. Adenosine kinase (ADK) is thought to be a primary route of adenosine metabolism because its pharmacological inhibition leads to increased adenosine levels in vitro. Therefore, reduced levels of ADK may lead to an increase of endogenous adenosine in the brain. To further investigate the role of adenosine in sleep we exploited a mouse model with a disruption of the Adk gene (the Adk knock-out mice do not survive after 14 days postnatal). Methods: Male Adk+/- mice $(n=15)$ and wild-type (WT) littermates $(n=14)$ were used in the study. Sleep was continuously recorded during a 24-h baseline followed by $6 \mathrm{~h}$ sleep deprivation (SD) and $18 \mathrm{~h}$ recovery. The effects of the ADK inhibitor, 5'-iodotubercidin (ITU; 0.75-1.5 mg/kg i.p.), on sleep, EEG spectra and brain temperature were evaluated in a cross-over design with vehicle. Results: During baseline the amount and distribution of waking, NREM sleep or REM sleep and their corresponding EEG power did not differ between Adk+/- and WT mice. SD induced a similar marked enhancement of NREM sleep amounts and slow-wave activity (SWA) in NREM sleep above baseline levels in both genotypes during the first $6 \mathrm{~h}$ recovery. No genotype difference was observed in the slow-wave energy in NREM sleep at the beginning of recovery or $18 \mathrm{~h}$ later. ITU induced mild hypothermia lasting approximately $1-2 \mathrm{~h}$ concomitant with a prominent reduction in EEG power in most frequencies in waking and NREM sleep during the first $2 \mathrm{~h}$ post-injection. SWA failed to be enhanced during $9 \mathrm{~h}$ following ITU treatment. Conclusion: Sleep and sleep regulation were not affected by the reduced expression levels of ADK, indicating compensatory mechanisms. Different kinetics of the processes underlying the build-up of sleep pressure and of ITU metabolism as well as hypothermia may have contributed to the lack of SWA increase after temporary inhibition of ADK.

Supported by SNSF grant nr. 3100A0-112528/1 and EU LSHM-CT-2005518189.

35

Time Course of Frontal Low EEG Activity During Sustained Wakefulness in Young Depressive Women. Is There an S-deficiency?

\section{A. Birchler-Pedross, S. Frey, P. Brunner, T. Götz, \\ V. Knoblauch, C. Cajochen \\ Centre for Chronobiology, Psychiatric University Clinics, Basel, Switzerland}

Aims: In an ongoing study we aim at investigating whether women suffering from major depression show impairments in the regulation of sleewake homeostasis (process S) and circadian rhythms. Here we focus on a marker of process $S$ during wakefulness. Thus, we quantified the time course of frontal low-EEG activity (FLA) and subjective sleepiness during a 40-h episode of sustained wakefulness in depressed in comparison to healthy control women. Methods: Depressive volunteers full field the DSM-IV criteria of a major depression assessed by a SKID Interview. Twelve EEG channels were recorded continuously in 6 young depressive ( 26 mean age $+/-5$ sd) and 8 young healthy women (25.33mean age+/-4.03sd) during a 40-h sleep deprivation protocol under constant routine conditions. The Karolinska drowsiness test was carried out in 1.25-h intervals for 3 minutes in order to gather artefact free EEG samples. The EEGs were offline manually scored for artefacts and subjected to spectral analysis. Subjective sleepiness was assessed using the Karolinska Sleepiness Scale every 30 min during the 40 -h of sustained wakefulness. Results: We observed a significantly faster build-up of frontal-low EEG activity (FLA, 1-4.5 Hz) during the first 16 hours of the CR (group $x$ time, $p<0.01$ ). Post-hoc analysis yielded significantly more FLA during the late evening in depressed than healthy women $(\mathrm{p}<0.03)$. This was paralleled by higher sleepiness ratings during this time window $(\mathrm{p}<0.05)$. Conclusion: Rather than an S-deficiency, we have preliminary evidence for a faster increase of sleep homeostatic pressure (process S) during wakefulness in depressed women. This could reflect a use-dependent phenomenon (enhanced rumination in depression), since it was only observed in frontal brain regions. Alternatively, it could be the consequence of a less efficient circadian arousal signal in the evening, thus allowing more intrusion of delta in frontal brain areas.

This Research was supported by Swiss National Science Foundation.

36

\section{Prostaglandin D Synthase: The First Biomarker Distinguishing Excessive Daytime Sleepiness from Fatigue?}

U. Kallweit ${ }^{1}$, K. Aritake ${ }^{2}$, Y. Urade ${ }^{2}$, M. Uh/1 , C.L. Bassetti', C.R. Baumann ${ }^{1}$

${ }^{1}$ University Hospital Zurich, Department of Neurology, Zurich, Switzerland, ${ }^{2}$ Osaka Bioscience Institute, Molecular Behavioral Biology, Osaka, Japan

Background/Aims: Endogeneous sleepromoting substances such as prostaglandins are suggested to be involved in homeostatic sleewake regulation. Lipocalin-type prostaglandin D synthase (L-PGDS) catalyzes the production of somnogenic prostaglandin $\mathrm{D}$, and appears to be decreased in the cerebrospinal fluid (CSF) of patients with excessive daytime sleepiness (EDS). It is, however, not known whether CSF L-PGDS levels are decreased also in patients with fatigue. We aimed at measuring CSF L-PGDS levels in relation to the presence of EDS and fatigue. Methods: In this prospective study, we included consecutive patients with neurological disorders, and with a clinical indication to perform a lumbar puncture. Patients with primary sleewake disorders (e.g. narcolepsy) were excluded from this analysis. We performed all lumbar punctures between $10 \mathrm{a} . \mathrm{m}$. and $1 \mathrm{p} . \mathrm{m}$. Thereafter, CSF was immediately frozen at $-80^{\circ} \mathrm{C}$ and sent to Japan for L-PGDS 
determination by ELISA. We analyzed data from 15 patients ( 9 women), mean age was 43 years (range 22-61). For the assessment of EDS and fatigue, we administered validated questionnaires (Epworth sleepiness scale; ESS, and fatigue severity scale; FSS). Results: Six of 15 subjects suffered from EDS (ESS $>10$ ), 8 subjects from fatigue (FSS $>4.0)$. EDS patients had lower L-PGDS levels $(16.3 \pm 3.4 \mu \mathrm{g} / \mathrm{mL})$ than non-EDS patients $(22.6 \pm 7.5 \mu \mathrm{g} /$ $\mathrm{mL}, \mathrm{p}=0.048)$. On the other hand, levels were similar between patients with $(20.2 \pm 9.0 \mu \mathrm{g} / \mathrm{mL})$ and without fatigue $(19.9 \pm$ $3.3 \mu \mathrm{g} / \mathrm{mL}, \mathrm{p}=0.94)$. An association between CSF L-PGDS and gender or age was not found. Conclusion: The first preliminary results of this ongoing bicenter study indicate L-PGDS may be the first biomarker differentiating EDS from fatigue.

\section{7}

\section{A Functional Genetic Variation of Adenosine Deaminase Modulates Waking Performance Irrespective of Self-rated Sleep Duration}

\author{
V. Bachmann"1,2, F. Klaus', S. Bodenmann'1, P. Brugger ${ }^{2,3}$, \\ H.P. Landolt ${ }^{1,2}$ \\ ${ }^{1}$ Institute of Pharmacology and Toxicology, University of \\ Zurich, ${ }^{2}$ Zurich Center for Integrative Human Physiology, \\ University of Zurich, ${ }^{3}$ Department of Neurology, University \\ Hospital of Zurich, Switzerland
}

Accumulating evidence suggests that the neuromodulator adenosine contributes to homeostatic sleewake regulation. In humans, a functional polymorphism of the gene encoding the adenosine metabolizing enzyme, adenosine deaminase (ADA), predicts duration and intensity of slow wave sleep in healthy individuals (Rétey et al., 2005). The impact of this polymorphism on waking performance is unknown. Alleles and genotypes of the $22 \mathrm{G}>\mathrm{A}$ polymorphism of ADA were determined in 242 males $(n=124)$ and females $(\mathrm{n}=118)$. All subjects (age range: $18-40$ years) filled in questionnaires about sleewake habits, and their performance on tasks of executive functioning, attention and memory were systematically quantified. In subsequent studies, individual carriers of the variant allele (G/A genotype) were prospectively compared to homozygous $\mathrm{G}$ allele carriers. Self-rated sleep duration as estimated with the Munich Chronotype Questionnaire did not differ between ADA genotypes. By contrast, counting tendency on a random number generation $(\mathrm{RNG})$ task was higher in women with $\mathrm{G} / \mathrm{A}(\mathrm{n}=14)$ than $\mathrm{G} / \mathrm{G}(\mathrm{n}=87)$ genotype. No significant effect was observed in men. However, adjacency on the RNG and overall performance on a d2-attention task were worse in 28 individuals (13 men, 15 women) with G/A genotype compared to individually age- and sex-matched subjects with $\mathrm{G} / \mathrm{G}$ genotype. Heterozygous carriers of the A allele $(n=10)$ showed higher subjective sleepiness (Stanford Sleepiness Scale) after one night without sleep, and performed worse than matched individuals with $\mathrm{G} / \mathrm{G}$ genotype on the psychomotor vigilance task throughout prolonged wakefulness. Our findings demonstrate that a functional polymorphism of ADA predicts waking functions in healthy adults. They further support the notion that adenosinergic mechanisms play an important role in sleep homeostasis. Together with our previous sleep and sleep
EEG data (Rétey et al., 2005), they suggest that individuals with genetically reduced adenosine deaminase activity (G/A-genotype) are under higher sleep pressure than subjects with unimpaired adenosine metabolism (G/G-genotype).

Research supported by Swiss National Science Foundation and Zürich Center for Integrative Human Physiology.

\section{8 \\ Per3 Polymorphism Predicts Physiological and Cognitive Performance Response to Sleep Loss}

A.U. Viola

Center for Chronobiology, Psychiatric University Clinics

Basel, Switzerland

Aims: To investigate the physiological consequences of the PER3 polymorphism. Methods: Healthy volunteers were selected on the basis of their genotype, irrespective of diurnal preference or sleep related characteristics. Approximately 400 volunteers (age 20-35) were genotyped for the PER3 polymorphism. Individuals homozygous for the rarer 5-repeat VNTR (PER3-5/5) and for the 4-repeat VNTR (PER3-4/4) were identified and matched for age, gender, ethnicity and body mass index. Twenty-eight subjects wore actigraphs and completed sleep diaries for three weeks prior to the five-day laboratory study. The“ in-lab" part of this study was completed by 24 participants and comprised two baseline sleep episodes followed by 40 -h sleep deprivation protocol under constant routine conditions, with a subsequent recovery sleep episode. Results: Sleep diaries and actigraphy analyses indicated that the two groups did not differ with respect to their habitual sleewake timing and sleep duration. Similarly, the two groups did not differ with respect to the timing of markers of hormonal rhythms, such as melatonin and cortisol, and on a novel circadian marker, PER3 mRNA. On the other hand, comparison of sleep structure between PER3-5/5 and PER3-4/4 subjects yielded significant differences: PER3-5/5 subjects showed more deep slow wave sleep during NREM sleep, more alpha activity in REM sleep, and, under sleep deprivation, they experienced higher sleep pressure. Furthermore, their cognitive performance on tasks that engage more frontal cortical areas, such as executive function tasks, was significantly deteriorated in comparison to PER3-4/4 participants, particularly during the biological night. Analyses of wake and sleep ECG data revealed a loss of parasympathetic control on autonomic balance in PER35/5 in comparison to PER3-4/4 subjects. Conclusions: While the clock gene PER3 does not appear to have a substantial effect on the circadian phase; it appears to affect several aspects of sleep homeostasis. The main implication is that sleep homeostasis can be affected by clock genes and that these effects may account for the inter-individual differences in the susceptibility to sleep loss and circadian phase misalignment, which can happen during jet lag and shift work. 
39

The Effects of Gamma-Hydroxybutyrate on Vigilance States and EEG: Role of GABAB Receptors

\author{
J. Vienne 1 , M. Gassmann², B. Bettler' ${ }^{2}$ M. Tafti ${ }^{1}$ \\ ${ }^{1}$ Center for Integrative Genomics (CIG), University of \\ Lausanne, Lausanne, ${ }^{2}$ Pharmazentrum, Department of \\ Biomedicine, Institute of Physiology, University of Basel, \\ Switzerland
}

Introduction: Gamma-hydroxybutyrate (GHB) and its precursor gamma-butyrolactone (GBL) have wide-range effects on vigilance and behavior. However, the mode of action of GHB remains unclear. Evidence indicates that GHB acts mainly through GABAB receptors that consist of two subunits; $G A B A B(1)$ and $-\mathrm{B}(2)$. GABAB(1) has two isoforms; $1 \mathrm{a}$ and $1 \mathrm{~b}$. To elucidate the mechanisms by which GHB affects vigilance state and the role of GABAB receptor subunits, the electroencephalogram (EEG) of different $\mathrm{GABAB}$ receptor subunit knock-out (KO) mice was studied before and after GBL administration. Methods: Adult male wild-type (WT) BALB/c, GABAB(1a) -/-, GABAB(1b) -/-, GABAB(1) -/- and $\operatorname{GABAB}(2)$-/- mice were implanted with EEG and EMG electrodes. Mice were recorded during $24 \mathrm{hr}$ baseline followed by daily injections with saline and 3 doses of GBL (50, 100 and $150 \mathrm{mg} / \mathrm{kg}$ i.p.). Results: Under baseline conditions, GABAB(1) -/-, GABAB(2) -/- and GABAB(1a) -/- mice exhibited spontaneous seizures. Sleep amount, sleep quality and the EEG were differentially affected in the different KO mice. GBL induced EEG slow waves $(1-4 \mathrm{~Hz})$ with a spike-like EEG pattern at high doses in $\operatorname{GABAB}(1 \mathrm{~b})$-/-, GABAB(1a) -/- and WT mice, but no behavioral or EEG changes were observed in mice without any functional GABAB receptors $(\mathrm{GABAB}(1)$-/and - $\mathrm{B}(2)-/-$ mice). The duration of the 'GBL-induced state' and the prevalence of EEG slow-waves (delta power) during this state increased with dose in each genotype. GABAB(1a) -/- mice showed 2-fold higher values of delta power than the 2 other genotypes. Although GBL suppressed sleep dose-dependently after injection and induced a dramatic but non-physiological high delta activity, none of the 3 genotypes clearly compensated this sleep loss and/or modified the kinetics of delta power. Conclusion: Our results demonstrate that $\mathrm{GABAB}(1 \mathrm{a})$ plays an important role in epilepsy, sleep and thalamcortical synchronization. GBL acts only through GABAB receptors to induce behavioral and EEG effects. The lack of a compensatory response to the sleep time lost due to GBL indicates that sleep need does not change during the GBL-induced state. Moreover, the slow waves that accompany this state do not seem to lower subsequent sleep need. Thus, the homeostatic regulation of sleep seems temporarily suspended during the GBL-induced state and the delta activity induced by GBL might functionally be different from delta activity expressed during 'physiological' sleep.

\section{0 \\ Subjective Sleep Measures in Pregnant Depressed and Non-depressed Women}

\author{
A. Bader ${ }^{1,2}$, A. Riecher-Rössler ${ }^{2}$, U. Frisch ${ }^{2}$, A. Wirz-Justice ${ }^{1}$ \\ ${ }^{1}$ Center for Chronobiology, Psychiatric University Clinics \\ Basel, ${ }^{2}$ Psychiatric Outpatient Department, University \\ Hospital Basel, Switzerland
}

Objective: Affective disorder during pregnancy is a common condition: one in ten pregnant women suffers from Major Depressive Disorder (MDD). One complaint in pregnancy is sleep disturbance, and this is also a characteristic of MDD. However, the literature about sleep disturbances in depressed pregnant women is sparse. This ongoing study compared subjective sleep in pregnant depressed and non-depressed women. Methods: Selfreported sleep measures were collected by 35 pregnant women with a DSM-IV diagnosis of MDD and 7 non-depressed pregnant women (CON). All women kept a diary for one week where they assessed daily, before bedtime, their feelings (relaxed-tense, somatically unwell-well, alert-sleepy, sated-hungry) and mood (worst-best) on a bipolar scale (VAS) and noted the next morning their sleep onset time, wake-up time, and nighttime awakenings (number, duration). Results: The two groups did not differ in age $(\mathrm{M}=32.4)$ and pregnancy week $(\mathrm{PW})(\mathrm{M}=20.6)$. Over all trimesters, they showed significant differences in $\operatorname{mood}(\mathrm{p}<.001)$, feeling tense $(p<.05)$, and duration of nighttime awakenings $(p<.05)$. Compared with CON, MDD showed in the second trimester worse mood $(\mathrm{p}<.05)$, and in the third trimester also lower mood $(\mathrm{p}<.05)$, longer duration of nighttime awakenings $(\mathrm{p}<.05)$, and a trend for longer sleep onset latency $(\mathrm{p}<.052)$. Within the groups, no significant difference between the second and third trimester was found. For all pregnant women, better mood correlated with less tension $(\mathrm{r}=-.466, \mathrm{p}<.05)$, better somatic wellbeing $(\mathrm{r}=.737$, $\mathrm{p}<.001)$, fewer $(\mathrm{r}=-.371, \mathrm{p}<.05)$ and shorter nighttime awakenings $(r=-.392, p<.05)$, and shorter sleep onset latency $(r=-.367$, $\mathrm{p}<.05$ ), when PW was controlled. For PW, no significant correlation was found. There were no significant correlations between the trimesters within the groups. Conclusion: Pregnant women with MDD had a significantly lower mood, higher tension, and more sleep disturbances than CON. Greater depressed mood in pregnancy was correlated with poorer somatic wellbeing, higher tension, more and longer nighttime awakenings, and prolonged sleep onset latency. There was no increase in sleep disturbances in the third compared with the second trimester. 
41

\section{Chronotypes and Sleewake Patterns in Children and their Parents}

\section{H. Werner, O.G. Jenni \\ Child Development Center, University Children's Hospital \\ Zurich, Switzerland}

Introduction: Sleewake patterns differ substantially among individuals. These inter-individual differences root in the genetically programmed circadian clock which determines the so called "chronotypes" (Aeschbach et al. 2003; Roenneberg et al. 2002). Up to now, there is very little known about the effects of chronotype differences within family members. How does parent-child adjustment occur when morning-oriented parents have a night-type child or the other way round? The fit between parents and children's chronotypes is an important factor in understanding and predicting conflicts in family relationships (Barber, 1994). We aimed to assess chronotypes and sleewake patterns in children and parents by subjective and objective methods, and to focus on the effects of differences between children and parents on parental distress. Methods: Chronotypes of healthy kindergarten children (age 5-7 years) were assessed by the Children's Chronotype Questionnaire (CCTQ, Werner et al., submitted 2008); parental chronotypes were assessed by the Munich Chronotype Questionnaire (MCTQ, Roenneberg, 2003). All subjects wore an actigraph (Acitwatch Plus AW4; Cambridge Neurotechnology, Cambridge, U.K.) and filled out a sleep diary over 8 consecutive days. Parental distress was examined with the short form of the Parental Stress Index (PSI-SF, Abedin, 1990); child's temperament was assessed by the Emotionality-Activity-Sociability Temperament Inventory (EAS, Buss \& Plomin, 1984). Results: 59 mother-child dyads were included in the study. Mean age of the children was 5.9 years (range $5-7$ years, $56 \%$ boys and $44 \%$ girls), mean age of the mothers 39.0 years (range 31-49 years). Children's and mother's chronotype as well as chronotype differences between children and mother was not associated with difficulties in parent-child interaction and perceived parental distress $(\mathrm{p}>.05)$. However, the later children went to bed on scheduled days $(r=.354, p=.006)$, the later the lights were switched off $(r=.376, p=.004)$ and the later children fell asleep $(r=.333, p=.011)$, the more stressed were the mothers through difficult interaction with the child (independent of children's age and temperament). Longer sleep latency $(r=.33$, $\mathrm{p}=.011)$ and longer time to get fully alert in the morning $(\mathrm{r}=.294$, $\mathrm{p}=.026)$ of the mothers as well as poorer self-rated sleep quality $(\mathrm{r}=-.488, \mathrm{p}=.000)$ was also associated with higher parental distress (total stress). Conclusion: Different chronotypes between parents and children may not directly cause difficulties in parentchild interaction. Difficult parent-child interactions and parental distress, however, are related to children's and parent's sleewake patterns. In line with Martin (2007), we suggest that parents of kindergarten children may adjust to child's chronotype over time, and conclude that sleewake patterns of parents and children are associated with perceived parental distress.

This study was supported by the Cramer Foundation and the Swiss National Science Foundation.

\section{2 \\ Sleep Quality in Renal Transplanted Recipients}

\author{
H. Burkhalter', S. Sereika², S. Engberg², A. Wirz-Justice ${ }^{3}$, \\ J. Steiger ${ }^{4}$, Sabina De Geest ${ }^{1}$ \\ ${ }^{1}$ Institute of Nursing Science, University of Basel, \\ ${ }^{2}$ Institute of Nursing science, University of Pittsburgh, Pa., \\ USA, ${ }^{3}$ Center for Chronobiology, Psychiatric University \\ Clinics Basel, ${ }^{4}$ Division of Transplant Immunology and \\ Nephrology, University Hospital Basel, Switzerland
}

Aim: Poor sleep quality (SQ) has been associated with poor clinical outcome in a number of chronically ill patient populations, but is not yet well studied in transplant patients. Assessing SQ in large cohort study such as the Swiss Transplant Cohort Study is therefore very relevant. The aim of this study was to asses the validity of 2 items: one to assess SQ and one daytime functioning (DF) item, using the Pittsburgh Sleep Quality Index (PSQI) as gold standard. We used the validation criteria proposed by the American Psychological Association (APA). Methods: We used a cross-sectional descriptive design, including a convenience sample of all adult renal transplants transplanted at the University Hospital of Basel in the last five years. The questionnaires (demographics, PSQI, HADS (depressive symptomatology), EQ5D (perceived health status)) and the SQ and DF items were mailed to the patients' homes. The SQ and DF item were analyzed separately and using their combined score (mean score). Data was analysed following the APA validity criteria: content validity (using the content validity index), response processes (frequency analysis), internal structure (correlation with the gold standard PSQI) and relation to other variables (hypotheses-testing from literature review). Results: The study included 156 renal transplanted recipients (29\% women), aged from 18 to 76 (Me: $52.32, \mathrm{SD}<.11 .86), 68 \%$ were married or were cohabiting. A third of the sample was transplanted within the last year (Me: 2,3 years, SD 1,57). The prevalence of poor sleep quality was $48.4 \%$ (Me: 6,61, SD 4,16) based on the PSQI and $30.7 \%$ using the SQ item $($ cut off $<6)(\mathrm{Me}: 7.14, \mathrm{SD} 2,09)$ and $34.1 \%$ reported poor daytime functioning (cut off $<6)(\mathrm{Me} 7.14$, SD 2.17). Content validity was good for the SQ item (Index: .81) and poor for the DF item (Index: .45), 2,5\% of the SQ and 3,7\% of DF were missing, showing adequate validity on responce process. Both the SQ and DF scores were negatively skewed, but no floor effect. A significant correlation was found between the PSQI and the SQ Item (p: $-.737 \mathrm{p}<.01)$, the DF Item $(\mathrm{p}:-.527 \mathrm{p}<.01)$ and the combined SQ/ DF score $(p:-.725 p<.01)$ indicating accetable validity in view of internal structure. Poor SQ, poor DF and the combined score showed a significant association with higher depressive symptomatology (p: SQ -.486 p<.01; DF -.668 p<.01; SQ/DF -.668 $\mathrm{p}<.01$ ), lower perceived health status on the EQ-5D (p: SQ -.420 $\mathrm{p}<.01$; DF -.618 $\mathrm{p}<.01$; SQ/DF -.592 $\mathrm{p}<.01)$ and lower subjective helath status on the visual analogue scale (p: SQ -.419 $\mathrm{p}<.01$; $\mathrm{DF}-.749 \mathrm{p}<.01 ; \mathrm{SQ} / \mathrm{DF}-.661 \mathrm{p}<.01)$ demonstrating validity in 
relation to other variables. Conclusion: The SQ item is a valid item to be used in cohort study for a general sleep quality screening. Based on validity testing the construct of daytime functioning is important, but the wording of this item needs to be improved.

This study is supported by a research grant from the Alfred und Erika BärSpychiger-Stiftung.

43

\section{Positional Therapy for Obstructive Sleep Apnea: Do Your Patients Use It at Home?}

\author{
R. Heinzer, V. Rey, G. Lecciso, J. Vodoz, C. Pellaton,
} A. Delessert, CH. Manzini, M. Tafti, G. Lavigne

Centre d'investigation et de recherche sur le sommeil (CIRS), Centre Hospitalier Universitaire Vaudois, Lausanne, Switzerland

Introduction: Positional therapy preventing patients from sleeping supine has been used for many years to treat positional obstructive sleep apnea (OSA). However, the actual usage and efficacy of this therapy have never been objectively assessed. Methods: Ten patients with positional OSA, who refused or could not tolerate CPAP were identified. Nine of these patients, in whom the efficacy of a positional therapy device was confirmed by an overnight study, were instructed to use it every night for 3 months. Nightly usage of the device during this 3 months period was monitored by an actigraph (Actiwatch) placed inside the positional device (patients were informed). A third night study was performed at the end of the 3 months period. Results: During the diagnostic night study, mean apnea-hypopnea index (AHI) was $24.2 \pm 10$ events/h $(49.7 \pm 21.8$ supine and $6.5 \pm 6.6$ nonsupine), with an average of $48.6 \pm 30 \%$ of the night spent supine. During the night study with the positional therapy, mean AHI was reduced to $5.7 \pm 3.2$ events $/ \mathrm{h}(\mathrm{p}=0.0018)$ and the $\%$ of time spent supine dropped to $8.1 \pm 8.4 \%(\mathrm{p}=0.011)$. Two of the nine patients stopped using the positional device, one because of a back pain and another because of a broken clavicle. Overall the positional device was worn $63 \pm 34 \%$ of the night (range $9.1-96.6 \%$ ) for an average of $8.2 \pm 2.3$ hours per night. Six of the seven patients who were still using the device at the end of the study had a third night study with the device: Mean AHI was 5.8 \pm 4.1 events per hour with $4.5 \pm 5.9 \%$ of the night spent supine. Conclusion: Usage of a positional therapy for OSA over a 3 months period is comparable to the reported CPAP usage and its efficacy persists at the end of this period.

Lausanne University young researcher grant. Swiss Pulmonary Society grant for research.

\section{4 \\ Oximetry Alone Versus Portable Polygraphy for Sleep Apnoea Screening Before Bariatric Surgery}

\author{
M. Malbois, M. Suter, C. Pellaton, J. Vodoz, V. Giusti, \\ R. Heinzer \\ University of Lausanne, Lausanne, Switzerland
}

Background: Bariatric surgery is now recognized as the only effective treatment for the morbidly obese patients. Obstructive sleep apnea (OSA) is a commonly encountered comorbidity in morbidly obese patients undergoing bariatric surgery. Screening for OSA is thus recommended as part of the preoperative assessment of these patients. The objective of this study was to compare the sensitivity of oximetry alone versus portable polygraphy in the preoperative screening for obstructive sleep apnea. Methods: We retrospectively reviewed polygraphic data recorded as part of the preoperative assessment before bariatric surgery from 68 consecutive morbidly obese patients between November 2006 and June 2008. Sleep apnea screening was performed with an Embletta system. The pulse oxymeter (Nonin, Plymouth, MN, USA) had a sampling rate of $10 \mathrm{~Hz}$. For each patient we compared the sensitivity of $3 \%$ or $4 \%$ desaturation index (oximetry alone) with apnea hypopnea index (polygraphy) to diagnose OSA and classify the patients as normal ( $<5$ events/h), mild (5-15 events/h) moderate (16-30 events/h) or severe ( $>30$ events/h). Results: Using apnea hypopnea index, $17,6 \%$ of the patients were classified as severe, $17,6 \%$ as moderate, $36,8 \%$ as mild and $28 \%$ as normal. Using $3 \%$ desaturation index, $22,1 \%$ of the patients were classified as severe, $29,4 \%$ as moderate, $38,2 \%$ as mild an $10.3 \%$ as normal. With $4 \%$ desaturation index, $20,6 \%$ were classified as severe, $10,3 \%$ as moderate, $39,7 \%$ as mild and 29.4 as normal. Overall, 4\% desaturation index as compared to AHI yielded a $95 \%$ negative predictive value to rule out obstructive sleep apnea $(\mathrm{AHI}<5 / \mathrm{h})$ and a $100 \%$ sensitivity $(0.85$ positive predictive value) to detect severe OSA (AHI > 30). Conclusion: Using oximetry with $4 \%$ desaturation index as a screening tool for OSA could allow us to rule out significant OSA in almost a third of morbidly obese patients during preoperative work up for bariatric surgery. This widely available technique could accelerate preoperative work up of these patients. We hypothesize that this high sensitivity of oximetry is specific to morbidly obese patients due to a reduction of their lung volume, especially when they lay supine. Patients with a positive oximetry should however undergo further sleep investigations to confirm the diagnosis and patients complaining of daytime sleepiness should be referred to a sleep specialist when oximetry is negative. 
45

\section{Role of the GABAA Alpha4-Subtype in Sleep and Sleep Regulation}

\section{Z. Kovacs, I. Tobler, R. Winsky-Sommerer \\ Institute of Pharmacology and Toxicology, University of \\ Zurich, Switzerland}

GABA, the predominant inhibitory transmitter in the brain, and its $\mathrm{GABA}_{\mathrm{A}}$ receptors play an important role in sleep regulation. A vast repertoire of $\mathrm{GABA}_{\mathrm{A}}$ receptor subtypes has been identified. The alpha4-subtype shows a very discrete expression in the brain. Notably, it is highly expressed in the thalamic relay nuclei which play a key-role in generating patterns of electrical activity characteristic of vigilance states. These receptors show specific electrophysiological properties and pharmacological profile. We made use of $\mathrm{GABA}_{\mathrm{A}}$ alpha4 subunit-knockout $(\mathrm{KO})$ mice to study the role of this receptor subtype in sleep and sleep regulation. We performed continuous electroencephalogram (EEG) recordings (parietal and frontal EEG) under baseline conditions $(24 \mathrm{~h})$, followed by $6 \mathrm{~h}$ sleep deprivation and $18 \mathrm{~h}$ recovery ( $\mathrm{n}=9$ alpha4-KO mice versus $\mathrm{n}=8$ wild-type littermates). Sleep deprivation by gentle procedures is a well-established method to enhance sleep pressure and thereby uncover differences in sleep regulation which may not be evident under baseline conditions. Preliminary analyses of the EEG recordings showed no difference in time spent in waking, non-rapid eye movement (NREM) sleep and REM sleep during baseline. Spectral analysis of the baseline EEG showed a decrease in EEG power spectrum in NREM sleep in alpha4-KO mice, specifically in the frontal EEG, in frequencies between 9-15 $\mathrm{Hz}$ encompassing the spindle frequency band. In contrast, the waking and REM sleep EEG power spectra showed no differences between the genotypes. Further analyses are ongoing to evaluate the response of alpha4-KO mice to sleep deprivation. The specific decrease in EEG power density observed during NREM sleep in alpha4-KO mice suggests a role of the alpha4-subtype in generating brain oscillations associated with NREM sleep.

Forschungskredit (University of Zurich); EU MC grant MCRTN-CT-2004512362 .
46

\section{Persistent Vegetative and Minimally Conscious States: Assessment of SleeWake- Behavior}

S. J. Regel', S.P. Loughran 1, L. Buetler², M. Wieser', R. Riener ${ }^{3}$, P. Achermann ${ }^{1}$

${ }^{1}$ Institute of Pharmacology and Toxicology, University of

Zurich, ${ }^{2}$ HUMAINE Clinic Zihlschlacht, ${ }^{3}$ Sensory-Motor

Systems Lab, Institute of Robotics and Intelligent

Systems, ETH Zurich \& University of Zurich, Switzerland

Aims: The characteristics of sleewake behavior in patients with severe brain damage is poorly understood. Patients in so called "Persistent Vegetative State" (VS) appear awake though they do not show any sign of consciousness. Emerging from VS, patients may reach the "Minimally Conscious State" (MCS) in which minimal behavioral evidence of self or environmental awareness appears. The severe brain damage seen in these patients is generally accompanied by changes in electrical brain activity. Yet, analysis of the sleep and wake EEG in VS and MCS patients is lacking with only few case studies reported. Furthermore, large discrepancies in observations exist, and the methodology has suffered from several important weaknesses. One important methodological consideration is whether scoring based on standard criteria is appropriate due to the high EEG alteration caused by the damaged brain area. In view of these shortcomings and to improve future diagnosis and prognosis, there is an urgent need for systematic sleewake behavior assessment in these patients. Methods: Three $\sim 24 \mathrm{~h}$ polysomnographic recordings were collected in three-week intervals in the framework of an ongoing collaborative project in which VS and MCS patients are physically stimulated by stepping movements and verticalisation on the dynamic tilt table "Erigo" (Hocoma AG, Volketswil, Switzerland). Five patients ( 3 males, 2 females) between 47 and 57 years of age (mean \pm SD: $48.6 \pm 5.7 \mathrm{y}$ ) have been evaluated to date. Scoring rules were developed as scoring according to standard criteria was not appropriate. Results: In general, patients exhibited similar physiological patterns across all of their three $\sim 24$ h-recordings, however, a high variability between patients was observed. Although all patients appeared to have sleep and wake-like states, none showed a clear or typical sleewake pattern as seen in healthy people. Conclusions: Sleewake-like-behavior in PVS and MCS does not show a common pattern across patients and is different to that seen in a healthy population. Accordingly, scoring based on standard criteria seems to be inappropriate. More data is needed to establish specific scoring rules and further explore sleewake-like behavior in these patients. 


\section{Neuropsychobiology}

Achermann, P. 26, 27, 28, 30, 46

Adam, M. 32

Anders, D. 22

Archer, S. 20

Aritake, K. 36

Bachmann, V. 15, 37

Bader, A. 40

Balu, S. 29

Bassetti, C.L. 11, 16-19, 23, 25, 36

Baumann, C.R. 25, 36

Bersagliere, A. 28

Bettler, B. 39

Birchler-Pedross, A. 2, 35

Bloch, K.E. 6

Bodenmann, S. 15, 37

Brown, S.A. 8, 9

Brugger, P. 16, 37

Brunner, P. 2, 35

Bucher, H.U. 3

Buchmann, A. 24

Buetler, L. 46

Burkhalter, H. 42

Caflisch, J. 13

Cajochen, C. 2, 8, 9, 10, 20, 29, 35

Cam, E. 11

Carskadon, M. A. 26

Casali, K.R. 12

Chellappa, S.L. 12, 20

Curie, T. 1, 7

Czeisler, C.A. 4

De Geest, S. 42

Delessert, A. 43

Dietz, V. 21

Dijk, D.J. 20

Dorsaz, S. 7

Duffy, J.F. 4

Eckert, A. 8, 9, 10

Eggenberger, D. 19

Emmenegger, Y. 7

Engberg, S. 42

Flammer, J. 22

Fontijn, J. 3

Franken, P. 1, 7

Frey, S. 2, 29, 35

Frisch, U. 40

Gadient, S. 23

Gao, B. 11

Gassmann, M. 39

Gast, H. 14

Geiger, A. 27, 31, 33
Numbers refer to abstract numbers

Geissler, E. 15

Gerling, A. 34

Gip, P. 1

Giusti, V. 44

Gompper, B. 22

Götz, T. 2, 35

Greusing, S. 29

Guyer, C. 3

Haeger, H. 11

Hedel, H. Van 21

Hefti, K. 21

Heinzer, R. 43, 44

Heller, H.C. 1

Huber, R. 3, 16, 24, 31, 33

Hübner, A. 23

Izakovic, J. 9

Jaggi-Schwarz, K. 15

Jenni, O.G. 3, 13, 24, 27, 31, 33, 41

Kallweit, U. 36

Khatami, R. 16

Klaus, F. 37

Knoblauch, V. 2, 35

Kovacs, Z. 45

Krafft, K. 23

Kräuchi, K. 22

Kurth, S. 24, 31, 33

Landolt, H.P. 15, 21, 32, 37

Lavigne, G. 43

Lecciso, G. 43

Loughran, S.P. 46

Malbois, M. 44

Manzini, CH. 43

Maret, S. 7

Mathis, J. 14, 19

Meier, F. 10

Michael, N. 25

Molinari, L. 13

Mongrain, V. 1, 7

Montano, N. 12

Münch, M. 4

Nussbaumer, Y. 6

Oberholzer, M. 17, 18

Orgül, S. 22

Pagani, L. 8, 9, 10

Palchykova, S. 34

Pellaton, C. 43, 44

Porta, A. 12
Poryazova, R. 16, 17, 18

Pugin, F. 20

Regel, S. J. 46

Retey, J.V. 32

Rey, V. 43

Riecher-Rössler, A. 40

Riener, R. 46

Ringli, M. 24, 31, 33

Ronda, J.M. 4

Rothen, N. 29

Rummel, C. 14

Rusterholz, T. 30

Schindler, K. 14

Schuepfer, N. 6

Sereika, S. 42

Siclari, F. 17

Silva, E.J. 4

Skene, D.J. 8

Steiger, J. 42

Stoll, C. 15

Suter, M. 44

Tafti, M. 39, 43

Tarokh, L. 26

Tobaldini, E. 12

Tobler, I. 34,45

Uhl, M. 36

Ulrich, S. 6

Urade, Y. 36

Van Dongen, H.P.A. 30

Vienne, J. 39

Viola, A.U. 12, 20, 38

Vodoz, J. 43, 44

Wehrle, R. 32

Werner, H. 41

Werth, E. 16, 23, 25

Wieser, M. 46

Winsky-Sommerer, R. 5, 34, 45

Wirz-Justice, A. 40, 42

Zimmermann, R. 23

Zunzunegui, C. 11
KARGER

(C) 2009 S. Karger AG, Basel

Fax +41613061234

E-Mail karger@karger.ch

www.karger.com

Accessible online at:

www.karger.com/nps 